\title{
Communications in molecules: local and multi-configuration channels and their entropic descriptors of bond multiplicity and composition
}

\author{
Roman F. Nalewajski
}

Received: 20 June 2013 / Accepted: 29 July 2013 / Published online: 5 September 2013

(C) The Author(s) 2013. This article is published with open access at Springerlink.com

\begin{abstract}
The Orbital Communication Theory of the chemical bond, in which molecules are treated as information systems transmitting "signals" of electron allocations to Atomic Orbitals, is extended to cover the local resolution level of electron distributions and the Configuration-Interaction (CI, multi-determinantal) description of molecular states. These communication systems generate the information-theoretic measures of both the absolute and relative multiplicities of chemical bonds, as well as the bond covalent (communication-noise) and ionic (information-flow) components. The orbital/local communications via the CI ensembles of the occupied molecular orbitals in such generalized molecular states are investigated. Illustrative two-orbital model and its prototype Valence-Bond structures are examined in a more detail.
\end{abstract}

Keywords Chemical bonds · Communication systems - Covalent/ionic components Entropic bond multiplicities - Information theory - Local information propagation · Orbital communications

\section{Introduction}

The Information Theory (IT) [1-8] has been successfully applied to explore the electron probabilities and patterns of chemical bonds they generate in molecules, e.g., [9-21]. In Schrödinger's quantum mechanics the electronic state is determined by the system wave-function, the (complex) amplitude of the particle probability distribution, which carries the classical part of the overall information content. Both the electron density or its shape factor, the probability distribution determined by the wave-function modulus, and the system current distribution, related to the gradient

\footnotetext{
R. F. Nalewajski $(\varangle)$

Department of Theoretical Chemistry, Jagiellonian University,

R. Ingardena 3, 30-060 Cracow, Poland

e-mail: nalewajs@chemia.uj.edu.pl
} 
of the wave-function phase, ultimately contribute to the resultant (quantum) information content of molecular states. The former reveals the classical information content, while the latter determines its non-classical complement in the overall information measure $[9,10,20-23]$.

The non-classical information terms, due to the electron current (or the wavefunction phase), introduce a non-vanishing information source into the associated entropy/information continuity equation, which expresses a local balance of the resultant information density $[10,13]$. Since the extremum principles of these generalized information measures ultimately determine the molecular equilibria implied by the Schrödinger equation $[2,13]$, this quantum IT treatment of the molecular electronic structure is thus equivalent to the standard quantum-mechanical description. The vertical information principles [20-23], for the fixed electron density, were shown to closely parallel the familiar energy and entropy principles of the ordinary thermodynamics.

Elsewhere it has been argued that many classical problems of theoretical chemistry can be approached afresh using the IT perspective [9-21]. For example, the displacements of the classical information distribution in a molecule, relative to the promolecular reference consisting of its non-bonded constituent atoms, have been investigated [11-15,17-21,24-26] and the least biased partition of the molecular electron distributions into subsystem contributions, e.g., densities of bonded atoms, has been examined [11-13,27-34]. This IT approach has been shown to lead to the "stockholder" Atomsin-Molecules (AIM) of Hirshfeld [35]. These optimum density pieces can be derived from alternative global and local variational principles of IT. They have been also generalized in a related problem of the AIM partitioning of two-electron densities [11,32-34].

The spatial localization of specific chemical bonds present another challenging problem to be tackled by this novel treatment of molecular systems. Another diagnostic problem of the molecular electronic structure deals with the shell structure and electron localization in atoms and molecules. The non-additive Fisher information in the Atomic Orbital (AO) resolution has been recently used as the Contra-Gradience (CG) criterion for localizing the bonding regions in molecules [11-21,25-38], while the related information density in the Molecular Orbital (MO) resolution has been shown $[11,39]$ to determine the vital ingredient of the Electron-Localization Function (ELF) [40-42].

The Communication Theory of the Chemical Bond (CTCB), which uses the entropic descriptors of the molecular information (communication) channels in the AIM, orbital and local resolutions of the electron probability distributions, has also been developed [11-13,43-60]. The same bond descriptors have been used to provide the information-scattering perspective on the intermediate stages in the electron redistribution processes [61], including the atom "promotion" via the orbital hybridization [62], and the communication theory for the excited electron configurations has been developed [63]. Moreover, the phenomenological description of equilibria in molecular subsystems has been proposed [11,64-66], which formally resembles that developed in ordinary thermodynamics [67].

Entropic probes of the electronic structure have already provided attractive tools for describing the chemical bond phenomenon in information terms. For an exploration of the chemical bond multiplicities in the orbitally-resolved communication theory $[13,58-60,68-71]$ it is vital to examine how the input information is propa- 
gated between $\mathrm{AO}$, the typical basis functions used to describe the bonding (occupied) MO subspace. In fact, the molecular system can be regarded as an information system determined by the communication network of the electronic conditional probabilities, in which the elementary "units" relevant to the resolution level in question emit and/or receive the electron-allocation signals [13]. This classical information scattering and flow processes can be characterized by standard tools of the Shannon's theory of communication $[3,4,7,8,11-13]$, thus providing a novel class of descriptors of molecular "connectivities" between AIM.

In particular, the communication noise, measured by the network average conditional entropy (scattered information), reflects the AO indeterminism in a molecule, and hence also the electron delocalization effect synonymous with the chemical covalency concept. The complementary bond component, chemical iconicity, is similarly probed by the channel average mutual information (information flow) descriptor, which reflects the AO deterministic (localization) aspect of the probability propagation in a molecule. These two IT components complement each other: the more ionic (deterministic) is the molecular communication system, the less covalent (indeterministic) is its probability propagation in the given $\mathrm{AO}$ basis. This reflects a competition between these two bond components for the available basis functions.

The classical (probability) entropic probes have been applied to interpret the molecular electron distributions in chemical terms. These communication descriptors have been derived from the classical information channels determined by the conditional probabilities of the AO events in the stationary (non-degenerate) molecular state, for which the spatial-phase component, and hence also the associated probability current, both identically vanish. The truly quantum channel, capable of the communication interference [72], calls for the information system of the probability-amplitude propagation, with the scattering amplitudes then explicitly depending on phases of the emitting and monitoring complex event-states. Such an amplitude channel, corresponding to the quantum scattering between complex basis functions in the complex molecular state, still awaits a more thorough examination [21].

The IT approach introduces into the theory of electronic structure of molecular systems the novel entropy-representation [10-23], which complements the familiar energy-representation of the molecular quantum mechanics. Such a dual perspective parallels that known from the ordinary thermodynamics [67]. It establishes the equivalent energy and entropy/information principles governing the molecular equilibria, provides a new unifying perspective on molecular electronic structure, extends the variety of tools for probing chemical processes, and enriches the range of available descriptors of the bonding patterns in molecules. It increases our understanding of the classical (intuitive) chemical concepts, e.g., the identity of AIM, bond localization, sources and measures of bond-order, its covalent/ionic composition, etc. The novel, through-bridge mechanism of the (intermediate) orbital interactions in molecules has been identified [73-77], which complements the familiar through-space (direct) bond contributions. The IT approach also covers changes in the bond pattern effected by chemical reactions [78,79]. The equivalence of the vertical (densityconstrained) energy and entropy/information rules in quantum mechanics parallels that of the complementary energy and entropy principles of thermodynamics. 
In this work we intend to examine the local communications in molecules and their bond-multiplicity descriptors. We shall also attempt to generalize the AO formulation of CTCB, called the Orbital Communication Theory (OCT) [12,13,58-60,6871], into the multi-determinantal expansions of electronic states encountered in the Configuration-Interaction (CI) treatment. Previous one-determinantal MO approach, e.g., within the Hartree-Fock (HF) or Kohn-Sham (KS) Self-Consistent Field (SCF) MO theories, will be extended to cover the multi-determinantal states of CI theory. The molecular networks for the electronic information delocalization among basis functions, both classical (probability propagation) and quantum (amplitude scattering), will then also cover the CI MO-ensembles. This treatment extends the previous Natural Orbital (NO) analysis [45] of the homonuclear bond in $\mathrm{H}_{2}$ within the Shull model [80-83]. The information dissipation/flow phenomena will be investigated using both the local and CI orbital descriptions, and the bond multiplicity/composition descriptors in prototype Valence-Bond (VB) structures will be reexamined.

Throughout the article the following tensor notation is used: $A$ denotes a scalar quantity, $\boldsymbol{A}$ stands for the row- or column-vector, and $\mathbf{A}$ represents a square or rectangular matrix. The logarithm of the Shannon-type information measure is taken to an arbitrary but fixed base. In keeping with the custom in works on IT the logarithm taken to base 2 corresponds to the information measured in bits (binary digits), while selecting $\log =\ln$ expresses the amount of information in nats (natural units): 1 nat = 1.44 bits.

\section{Communication channels and their information descriptors}

We begin with some rudiments on the molecular information networks and the entropy/information descriptors of a transmission of the electron-assignment "signals" in molecular communication systems [11-13,46-63]. The basic elements of such a "device" are shown in Fig. 1. The signal emitted from $n$ "inputs" $\boldsymbol{a}=\left(a_{1}, a_{2}, \ldots, a_{n}\right)$ of the channel source $\mathbf{A}$ is characterized by the probability distribution $\boldsymbol{P}(\boldsymbol{a})=$ $\boldsymbol{p}=\left(p_{1}, p_{2}, \ldots, p_{n}\right)$. It can be received at $m$ "outputs" $\boldsymbol{b}=\left(b_{1}, b_{2}, \ldots, b_{m}\right)$ of

Input (Source): A Communication network: $\mathbf{P}(\boldsymbol{b} \mid \boldsymbol{a}) \quad$ Output (Receiver): $\mathbf{B}$

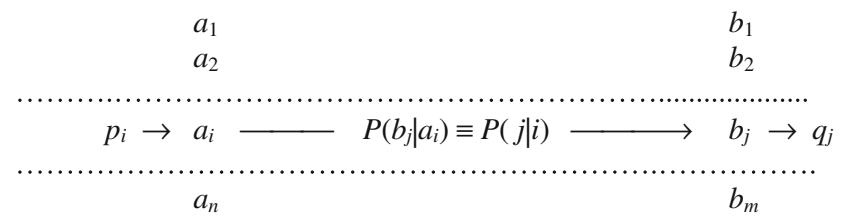

Fig. 1 Schematic diagram of the communication system characterized by two probability vectors: $\boldsymbol{P}(\boldsymbol{a})=$ $\left\{P\left(a_{i}\right)\right\}=\boldsymbol{p}=\left(p_{1}, \ldots, p_{n}\right)$, of the channel "input" events $\boldsymbol{a}=\left(a_{1}, \ldots, a_{n}\right)$ in the system source $\mathbf{A}$, and $\boldsymbol{P}(\boldsymbol{b})=\left\{P\left(b_{j}\right)\right\}=\boldsymbol{q}=\left(q_{1}, \ldots, q_{m}\right)$, of the "output" events $\boldsymbol{b}=\left(b_{1}, \ldots, b_{m}\right)$ in the system receiver B. The transmission of signals in this communication channel is described by the $(n \times m)$-matrix of the conditional probabilities $\mathbf{P}(\boldsymbol{b} \mid \boldsymbol{a})=\left\{P\left(b_{j} \mid a_{i}\right) \equiv P(j \mid i)=P(i, j) / p_{i}\right\} \equiv \mathbf{P}(\boldsymbol{q} \mid \boldsymbol{p})$ of observing different "outputs" (columns, $j=1,2, \ldots, m)$, given the specified "inputs" (rows, $i=1,2, \ldots, n)$. For clarity, only a single scattering $a_{i} \rightarrow b_{j}$ is shown in the diagram 
the system receiver $\mathbf{B}$. The transmission of signals in the channel is randomly disturbed thus exhibiting a communication noise. The signal propagation is described by the conditional probabilities of the "outputs-given-inputs", $\mathbf{P}(\boldsymbol{b} \mid \boldsymbol{a})=\left\{P\left(b_{j} \mid a_{i}\right)=\right.$ $\left.P\left(a_{i} \wedge b_{j}\right) / P\left(a_{i}\right) \equiv P(j \mid i)\right\} \equiv \mathbf{P}(\boldsymbol{q} \mid \boldsymbol{p})$; here $\mathbf{P}(\boldsymbol{a}, \boldsymbol{b})=\left\{P\left(a_{i} \wedge b_{j}\right) \equiv P(i, j)\right\}$ groups probabilities of a joint occurrence of the specified pair of the input and output events. The relevant normalization conditions of these probabilities read:

$$
\begin{aligned}
& \sum_{j} P(i, j)=p_{i}, \quad \sum_{i} P(i, j)=q_{j}, \\
& \sum_{i} \sum_{j} P(i, j)=1 ; \quad \sum_{j} P(j \mid i)=1, \quad i=1,2, \ldots
\end{aligned}
$$

The distribution of the output signal $\boldsymbol{P}(\boldsymbol{b})=\boldsymbol{q}$ among the detection "events" $\boldsymbol{b}$ is given by the output probability distribution $\boldsymbol{q}=\left(q_{1}, q_{2}, \ldots, q_{m}\right)=\boldsymbol{P}(\boldsymbol{a}) \mathbf{P}(\boldsymbol{b} \mid \boldsymbol{a})=\boldsymbol{p} \mathbf{P}(\boldsymbol{q} \mid \boldsymbol{p})$.

The Shannon entropy of the "product" distribution $\mathbf{P}(\boldsymbol{a}, \boldsymbol{b})$ can be expressed as the sum of the average entropy in the marginal (input) probability distribution $\boldsymbol{p}$,

$$
S(\boldsymbol{p})=-\sum_{i} p_{i} \log p_{i}
$$

and the average conditional entropy in $\boldsymbol{q}$ given $\boldsymbol{p}$ (see Fig. 2),

$$
\left.S(\boldsymbol{q} \mid \boldsymbol{p})=-\sum_{i} \sum_{j} P(i, j) \log P(j \mid i)\right],
$$

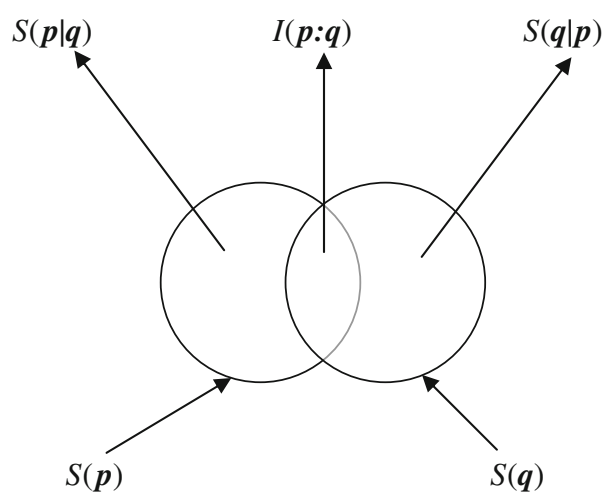

Fig. 2 Diagram of the conditional-entropy and mutual-information quantities for two dependent probability distributions $\boldsymbol{p}$ and $\boldsymbol{q}$ of Fig. 1. Two circles enclose areas representing the entropies $S(\boldsymbol{p})$ and $S(\boldsymbol{q})$ of two separate probability vectors/schemes, while their common (overlap) area corresponds to the mutual information $I(\boldsymbol{p}: \boldsymbol{q})$ in these two distributions. The remaining part of each circle represents the corresponding conditional entropy, $S(\boldsymbol{p} \mid \boldsymbol{q})$ or $S(\boldsymbol{q} \mid \boldsymbol{p})$, measuring the residual uncertainty about events in one set, when one has the full knowledge of the occurrence of events in the other set of outcomes. The area enclosed by the envelope of two circles thus represents the entropy of the "product" (joint) distribution: $S(\mathbf{P}(\boldsymbol{a}, \boldsymbol{b}))=$ $S(\boldsymbol{p})+S(\boldsymbol{q})-I(\boldsymbol{p}: \boldsymbol{q})=S(\boldsymbol{p})+S(\boldsymbol{q} \mid \boldsymbol{p})=S(\boldsymbol{q})+S(\boldsymbol{p} \mid \boldsymbol{q})$ 


$$
\begin{aligned}
S[\mathbf{P}(\boldsymbol{a}, \boldsymbol{b})] & =-\sum_{i} \sum_{j} P(i, j) \log P(i, j) \\
& =-\sum_{i} \sum_{j} p_{i} P(j \mid i)\left[\log p_{i}+\log P(j \mid i)\right] \\
& =-\left[\sum_{j} P(j \mid i)\right] \sum_{i} p_{i} \log p_{i}-\sum_{i} p_{i}\left[\sum_{j} P(j \mid i) \log P(j \mid i)\right] \\
& \equiv S(\boldsymbol{p})+S(\boldsymbol{q} \mid \boldsymbol{p}),
\end{aligned}
$$

The latter represents the extra amount of the information about the occurrence of events $\boldsymbol{b}$, given that the events $\boldsymbol{a}$ are known to have occurred. In other words: the amount of information obtained as a result of simultaneously observing the events $\boldsymbol{a}$ and $\boldsymbol{b}$ of two discrete probability distributions equals to the amount of information in one set, say $\boldsymbol{a}$, supplemented by the extra information provided by the occurrence of events in the other set $\boldsymbol{b}$, when $\boldsymbol{a}$ are known to have occurred already.

The common amount of information in two dependent events $a_{i}$ and $b_{j}, I(i: j)$, measuring the information about $a_{i}$ provided by the occurrence of $b_{j}$ or the information about $b_{j}$ provided by the occurrence of $a_{i}$, determines the mutual information in these two events:

$$
\begin{aligned}
I(i: j) & =\log \left[P(i, j) /\left(p_{i} q_{j}\right)\right]=\log \left[P(i \mid j) / p_{i}\right] \equiv \log \left[P(j \mid i) / q_{j}\right] \\
& =I(j: i) .
\end{aligned}
$$

It vanishes, when both events are independent, i.e., when the occurrence of one event does not influence (or condition) the probability of the occurrence of the other event, and it is negative, when the occurrence of one event makes a non-occurrence of the other event more likely. It also follows from the preceding equation that

$$
\begin{aligned}
& I(i: j)=I(i)-I(i \mid j)=I(j)-I(j \mid i)=I(i)+I(j)-I(i, j) \text { or } \\
& I(i, j)=I(i)+I(j)-I(i: j),
\end{aligned}
$$

where the self-information of the joint event $I(i, j)=-\log P(i, j)$. Thus, the information in the joint occurrence of two events $a_{i}$ and $b_{j}$ is the information in the occurrence of $a_{i}$ plus that in the occurrence of $b_{j}$ minus the mutual information. Clearly, for independent events, when $P^{i n d} \cdot(i, j)=p_{i} q_{j}, I^{i n d} \cdot(i: j)=0$ and hence $I^{i n d} \cdot(i, j)=I(i)+I(j)$.

The mutual information of an event with itself defines its self-information: $I(i$ : $i) \equiv I(i)=\log \left[P(i \mid i) / p_{i}\right]=-\log p_{i}$, since $P(i \mid i)=1$. It vanishes, when $p_{i}=1$, i.e., when there is no uncertainty about the occurrence of $a_{i}$, so that the occurrence of this event removes no uncertainty, hence conveys no information. This quantity provides a measure of the uncertainty about the occurrence of the event itself, i.e., the information received when this event actually occurs. The Shannon entropy of Eq. (2) can be thus interpreted as the mean value of self-informations $\left\{I(i)=-\log p_{i}\right\}$ in individual events: $S(\boldsymbol{p})=\sum_{i} p_{i} I(i)$. One similarly defines the average mutual 
information in two probability distributions (see Fig. 2) as the mean value of the mutual information quantities for individual joint events:

$$
\begin{aligned}
I(\boldsymbol{p}: \boldsymbol{q}) & =\sum_{i} \sum_{j} P(i, j) I(i: j)=\sum_{i} \sum_{j} P(i, j) \log \left[P(i, j) / P^{i n d} \cdot(i, j)\right] \\
& =S(\boldsymbol{p})+S(\boldsymbol{q})-S[\mathbf{P}(\boldsymbol{a}, \boldsymbol{b})]=S(\boldsymbol{p})-S(\boldsymbol{p} \mid \boldsymbol{q})=S(\boldsymbol{q})-S(\boldsymbol{q} \mid \boldsymbol{p}) \geq 0,
\end{aligned}
$$

where equality holds for independent distributions. Indeed, the amount of uncertainty in $\boldsymbol{q}$ can only decrease when $\boldsymbol{p}$ has been known beforehand, $S(\boldsymbol{q}) \geq S(\boldsymbol{q} \mid \boldsymbol{p})=$ $S(\boldsymbol{q})-I(\boldsymbol{p}: \boldsymbol{q})$, with equality being observed only when the two sets of events are independent, thus giving non-overlapping entropy circles in Fig. 2.

The average mutual information is also an example of the entropy deficiency (cross entropy, missing information, information distance, directed divergence) of Kullback and Leibler [5,6]. For example, in the discrete probability scheme identified by events $\boldsymbol{a}=\left\{a_{i}\right\}$ and their probabilities $\boldsymbol{P}(\boldsymbol{a})=\boldsymbol{p}$, this discrimination information in $\boldsymbol{p}$ with respect to the reference distribution $\boldsymbol{P}\left(\boldsymbol{a}_{0}\right)=\boldsymbol{p}_{0}=\left\{p_{i}^{0}\right\}$ reads:

$$
\Delta S\left(\boldsymbol{p} \mid \boldsymbol{p}_{0}\right)=\sum_{i} p_{i} \log \left(p_{i} / p_{i}^{0}\right) \equiv \sum_{i} p_{i} I_{i} \geq 0
$$

This quantity provides a measure of the information resemblance between the two compared probability schemes. The more the two distributions differ from one another, the larger this information distance. For individual events the logarithm of the probability ratio $I_{i}=\log \left(p_{i} / p_{i}^{0}\right)$, called the probability surprisal, provides a measure of the event information in the current distribution relative to that in the reference distribution. Notice that the equality in the preceding equation takes place only for the vanishing surprisals in all events, i.e., when the two probability distributions are identical.

Indeed, the average mutual information of Eq. (7) measures the missing information between the joint probabilities $\mathbf{P}(\boldsymbol{a}, \boldsymbol{b}) \equiv \boldsymbol{\pi}$ of the dependent events $\boldsymbol{a}$ and $\boldsymbol{b}$, and the joint probabilities $\mathbf{P}^{\text {ind. }}(\boldsymbol{a}, \boldsymbol{b})=\boldsymbol{\pi}_{0}=\boldsymbol{p}^{\mathrm{T}} \boldsymbol{q}$ for the independent joint events: $I(\boldsymbol{p}: \boldsymbol{q})=$ $\Delta S\left(\pi \mid \pi_{0}\right)$. The average mutual information thus reflects a degree of a dependence between events defining the two probability schemes. A similar information-distance interpretation can be attributed to the average conditional entropy of Eq. (3): $S(\boldsymbol{p} \mid \boldsymbol{q})=$ $S(\boldsymbol{p})-\Delta S\left(\boldsymbol{\pi} \mid \boldsymbol{\pi}_{0}\right)$.

\section{Orbital channels in a single electron configuration}

In OCT the orbital channels [3,4,6,11-13] propagate probabilities of electron assignments to basis functions of SCF MO calculations, e.g., Atomic Orbitals (AO) $\chi=$ $\left(\chi_{1}, \chi_{2}, \ldots, \chi_{m}\right)$. The underlying conditional probabilities of the output orbital events, given the input orbitals, $\mathbf{P}\left(\chi^{\prime} \mid \chi\right)=\left\{P\left(\chi_{j} \mid \chi_{i}\right) \equiv P(j \mid i) \equiv P_{i \rightarrow j}=A(j \mid i)^{2}=\right.$ $\left.\left(A_{i \rightarrow j}\right)^{2}\right\}$, or the associated scattering amplitudes $\mathbf{A}\left(\chi^{\prime} \mid \chi\right)=\left\{A(j \mid i)=A_{i \rightarrow j}\right\}$ of the emitting (input) states $\boldsymbol{a}=|\chi\rangle=\left\{\left|\chi_{i}\right\rangle\right\}$ among the monitoring/receiving (output) states $\boldsymbol{b}=\left|\chi^{\prime}\right\rangle=\left\{\left|\chi_{j}\right\rangle\right\}$, results from the (bond-projected) superposition principle of 
quantum mechanics [58-60,68-72,84]. The local description, which we shall examine in the present analysis, uses basis functions $\{|\boldsymbol{r}\rangle\}$ of the position representation identified by the continuous labels of spatial coordinates determining the location $\boldsymbol{r}$ of an electron. They determine both the input $\boldsymbol{a}=\{|\boldsymbol{r}\rangle\}$ and output $\boldsymbol{b}=\left\{\left|\boldsymbol{r}^{\prime}\right\rangle\right\}$ of the local molecular channel determined by the relevant conditional probabilities $\left\{P\left(\boldsymbol{r}^{\prime} \mid \boldsymbol{r}\right)=P_{\boldsymbol{r} \rightarrow \boldsymbol{r}^{\prime}}=\left(A_{\boldsymbol{r} \rightarrow \boldsymbol{r}^{\prime}}\right)^{2}\right\}$.

The entropy/information indices of the covalent/ionic components of chemical bonds represent the complementary descriptors of the average communication noise and of the amount of information flow, respectively, in the molecular orbital channel. One observes that the molecular input $\boldsymbol{P}(\boldsymbol{a}) \equiv \boldsymbol{p}$ generates the same distribution in the output of the molecular channel, $\boldsymbol{q}=\boldsymbol{p} \mathbf{P}(\boldsymbol{b} \mid \boldsymbol{a})=\left\{\sum_{i} p_{i} P(j \mid i) \equiv \sum_{i} P(i \wedge j)=\right.$ $\left.p_{j}\right\}=\boldsymbol{p}$, thus identifying $\boldsymbol{p}$ as the stationary vector of AO-probabilities in the molecular ground state. This purely molecular communication channel is devoid of any reference (history) of the chemical bond formation and generates the average noise index of the IT bond-covalency measured by the conditional-entropy of the system outputs given inputs: $S(\boldsymbol{P}(\boldsymbol{b}) \mid \boldsymbol{P}(\boldsymbol{a}))=S(\boldsymbol{q} \mid \boldsymbol{p}) \equiv S$.

The AO channel with the promolecular input signal, $\boldsymbol{P}\left(\boldsymbol{a}_{0}\right)=\boldsymbol{p}_{0}=\left\{p_{i}^{0}\right\}$, of to the system free constituent atoms, refers to the initial stage in the bond-formation process. It corresponds to the ground-state (fractional) occupations of the AO contributed by the system constituent atoms, before their mixing into Molecular Orbitals (MO). These input probabilities give rise to the average information flow index of the system IT bond-ionicity, given by the mutual-information in the channel promolecular inputs and molecular outputs [44]:

$$
\begin{aligned}
I\left(\boldsymbol{P}\left(\boldsymbol{a}_{0}\right): \boldsymbol{P}(\boldsymbol{b})\right) & =I\left(\boldsymbol{p}_{0}: \boldsymbol{q}\right)=\sum_{i} \sum_{j} P(i, j) \log \left[p_{i} P(i, j) /\left(p_{i} p_{j} p_{i}^{0}\right)\right] \\
& =\sum_{i} \sum_{j} P(i, j)\left[-\log p_{j}+\log \left(p_{i} / p_{i}^{0}\right)+\log P(j \mid i)\right] \\
& =S(\boldsymbol{q})+\Delta S\left(\boldsymbol{p} \mid \boldsymbol{p}_{0}\right)-S \equiv I_{0} .
\end{aligned}
$$

This amount of information reflects the fraction of the initial (promolecular) information content $S\left(\boldsymbol{p}_{0}\right)$ which has not been dissipated as noise in the molecular communication system. In particlular, for the molecular input, when $\boldsymbol{p}_{0}=\boldsymbol{p}$ and hence the vanishing information distance $\Delta S\left(\boldsymbol{p} \mid \boldsymbol{p}_{0}\right)=0$,

$$
I(p: q)=S(q)-S \equiv I
$$

The sum of these two bond components, e.g.,

$$
M\left(\boldsymbol{P}\left(\boldsymbol{a}_{0}\right) ; \boldsymbol{P}(\boldsymbol{b})\right)=M\left(\boldsymbol{p}_{0} ; \boldsymbol{q}\right)=S+I^{0}=S(\boldsymbol{q})+\Delta S\left(\boldsymbol{p} \mid \boldsymbol{p}_{0}\right) \equiv M_{0},
$$

measures the absolute overall IT bond-multiplicity, of all bonds in the molecular system under consideration, relative to the promolecular reference. For the molecular input this quantity preserves the Shannon entropy of the molecular input probabilities: 


$$
M(\boldsymbol{p} ; \boldsymbol{q})=S(\boldsymbol{q} \mid \boldsymbol{p})+I(\boldsymbol{p}: \boldsymbol{q})=S(\boldsymbol{q}) \equiv M .
$$

The relative index,

$$
\Delta M=M-M_{0}=\Delta S\left(\boldsymbol{p} \mid \boldsymbol{p}_{0}\right)
$$

reflecting changes due to the chemical bonds alone, is then interaction dependent. It correctly vanishes in the atomic dissociation limit of separated atoms, when $\boldsymbol{p}_{0}$ and $\boldsymbol{p}$ become identical. The entropy deficiency index $\Delta S\left(\boldsymbol{p} \mid \boldsymbol{p}_{0}\right)$, reflecting the information distance between the molecular electron density, generated by the constituent bonded atoms, and promolecular density, due to molecularly placed non-bonded constituent atoms, thus represents the overall IT difference-index of the system chemical bonds. Another interaction-dependent approach has been proposed within the Natural Orbital (NO) description of $\mathrm{H}_{2}$ [45].

An exploration of the chemical bond system in electronic states indeed calls for the $\mathrm{AO}$ resolution determined by the basis functions $\chi=\left(\chi_{1}, \chi_{2}, \ldots, \chi_{m}\right)$ of typical SCF LCAO MO calculations. The occupied MO of the familiar Hartree-Fock (HF) theory define the bonding subspace $\varphi^{0}=\chi \mathbf{C}^{0}\left\{\varphi_{s}^{0}=\phi_{s}^{0} \xi_{s}, s=1,2, \ldots, N\right\}$ of the (singly-occupied) spin-MO (SMO) in the molecular ground-state of $N$ electrons given by the Slater determinant consisting of $N$-lowest SMO:

$$
\Psi_{0}(N)=\operatorname{det}\left[\varphi^{0}\right] \equiv\left|\varphi_{1}^{0}, \varphi_{2}^{0}, \ldots, \varphi_{N}^{0}\right|
$$

Here, $\varphi_{s}^{0}(\boldsymbol{r}, \sigma)=\varphi_{s}^{0}(\boldsymbol{r}) \xi_{s}^{0}(\sigma), \phi_{s}^{0}$ denotes the spatial MO, and $\xi_{s}^{0}$ stands for one of two admissible spin states of an electron: $\xi_{s}^{0} \in\{\alpha($ spin-up), $\beta$ (spin-down) $\}$. One recalls at this point, that—rigorously speaking — in the Kohn-Sham (KS) theory such determinant of the method orbitals, which provides quite an adequate description of the system chemical bonds, e.g., [11-13], defines the hypothetical state of non-interacting electrons, which generates the same density as the (Coulomb-correlated) ground state of the real (interacting) system.

In this simplest, one-determinantal orbital approximation one thus takes into account only a single orbital configuration, e.g., the ground-state $\Psi_{0}(N)$, the occupied SMO of which give rise to all physical properties of the system under consideration. This configuration is thus uniquely identified by its singly-occupied (physical) SMO subspace $\varphi^{0}=\left(\varphi_{1}^{0}, \varphi_{2}^{0}, \ldots, \varphi_{N}^{0}\right)$, or by the associated spatial MO, $\phi^{0}=$ $\left(\phi_{1}^{0}, \phi_{2}^{0}, \ldots, \phi_{N}^{0}\right)=\chi \mathbf{C}^{0}$, which define the corresponding (idempotent) projectors:

$$
\hat{\mathrm{P}}_{\varphi}^{0} \equiv \sum_{s=1}^{N}\left|\phi_{s}^{0}\right\rangle\left\langle\phi_{s}^{0}|=| \varphi^{0}\right\rangle\left\langle\varphi^{0}\left|, \quad\left(\hat{\mathrm{P}}_{\varphi}^{0}\right)^{2}=\hat{\mathrm{P}}_{\varphi}^{0} ; \quad \hat{\mathrm{P}}_{\phi}^{0} \equiv\right| \phi^{0}\right\rangle\left\langle\phi^{0}\right|, \quad\left(\hat{\mathrm{P}}_{\phi}^{0}\right)^{2}=\hat{\mathrm{P}}_{\phi}^{0} .
$$

They generate the configuration Charge-and-Bond-Order (CBO) matrix, i.e., the oneelectron density matrix in the $\mathrm{AO}$ representation: 


$$
\boldsymbol{\gamma}^{0}=\left\langle\chi \mid \varphi^{0}\right\rangle\left\langle\varphi^{0} \mid \chi\right\rangle \equiv\left\langle\chi\left|\hat{\mathrm{P}}_{\varphi}^{0}\right| \chi\right\rangle=\mathbf{C}^{0} \mathbf{C}^{0 \dagger}=\left\{\gamma_{k, l}(0)\right\}, \quad \operatorname{tr} \boldsymbol{\gamma}^{0} \equiv \sum_{i} \gamma_{i},{ }_{i}(0)=N
$$

where the rectangular matrix of the LCAO MO expansion coefficients $\mathbf{C}^{0}=\left\langle\chi \mid \varphi^{0}\right\rangle=$ $\left\langle\chi \mid \phi^{0}\right\rangle$. For the complete AO basis, when $|\chi\rangle\langle\chi|=1$, the CBO matrix is also idempotent: $\left(\boldsymbol{\gamma}^{0}\right)^{2}=\boldsymbol{\gamma}^{0}$.

The conditional probabilities of $\mathrm{AO}$ communications in this ground-state configuration, via its occupied MO,

$$
\begin{aligned}
\mathbf{P}^{0}\left(\chi^{\prime} \mid \chi\right) & =\left\{P^{0}(j \mid i) \equiv P_{i \rightarrow j}(0)=A^{0}(j \mid i)^{2}=\left[A_{i \rightarrow j}(0)\right]^{2}\right. \\
& \left.=\gamma_{i, j}(0) \gamma_{j, i}(0) / \gamma_{i, i}(0)\right\},
\end{aligned}
$$

then determine the associated joint-probability matrix:

$$
\mathbf{P}^{0}\left(\chi, \chi^{\prime}\right)=\left\{P^{0}(i, j)=p_{i}(0) P^{0}(j \mid i)=\gamma_{i, j}(0) \gamma_{j, i}(0) / N\right\},
$$

where the configuration AO probabilities $\boldsymbol{p}^{0}=\left\{p_{i}(0)=\gamma_{i, i}(0) / N\right\}$. It can be straightforwardly verified that for the complete basis set they satisfy the normalization conditions of Eq. (1), e.g.,

$$
\sum_{j} P^{0}(i, j)=(1 / N) \sum_{j} \gamma_{i, j}(0) \gamma_{j, i}(0)=\gamma_{i, i}(0) / N=p_{i}(0) .
$$

The associated communication amplitudes then read:

$$
\mathbf{A}^{0}\left(\chi^{\prime} \mid \chi\right)=\left\{A^{0}(j \mid i)=A_{i \rightarrow j}(0)=\gamma_{i, j}(0) /\left[\gamma_{i, i}(0)\right]^{1 / 2}\right\} .
$$

They are dependent upon phases of the CBO matrix elements thus being capable of the communication "interference" $[72,85]$.

\section{Local communications in single electron configuration}

A deeper understanding of the molecular electronic structure ultimately calls for the (continuous) fine-grained, local description [86], to complement the (discrete) coarse-grained AO resolution adopted in OCT. One observes that the above orbitalcommunication development can indeed be naturally generalized into such an extreme resolution level of resolving the electron distributions in molecules, when one examines the information propagations between infinitesimal volume elements around $\boldsymbol{r} \in \Re$ in the channel input and $\boldsymbol{r}^{\prime} \in \Re^{\prime}$ in its output, respectively, where $\mathfrak{R}$ or $\Re^{\prime}$ denote the whole physical space.

As alredy mentioned in the preceding section, in such an approach one adopts the local basis set of the precise localization states $\{|\boldsymbol{r}\rangle\}$ of an electron, in which the MO projectors of Eq. (15) give rise to the ordinary (idempotent) one-electron density 
matrix. For example, for the single, say ground-state Slater determinant [Eq. (14)] in HF or KS theories one finds:

$$
\begin{aligned}
\gamma^{0}\left(\boldsymbol{r}, \boldsymbol{r}^{\prime}\right)=\left\langle\boldsymbol{r} \mid \boldsymbol{\phi}^{0}\right\rangle\left\langle\boldsymbol{\phi}^{0} \mid \boldsymbol{r}^{\prime}\right\rangle & =\left\langle\boldsymbol{r}\left|\hat{\mathrm{P}}_{\boldsymbol{\phi}}^{0}\right| \boldsymbol{r}^{\prime}\right\rangle=\sum_{s} \phi_{s}^{0}(\boldsymbol{r}) \phi_{s}^{0}\left(\boldsymbol{r}^{\prime}\right)^{*} \\
\operatorname{tr} \gamma^{0} \equiv \int \gamma^{0}(\boldsymbol{r}, \boldsymbol{r}) d \boldsymbol{r} & =\int \rho^{0}(\boldsymbol{r}) d \boldsymbol{r}=N \\
\int \gamma^{0}\left(\boldsymbol{r}, \boldsymbol{r}^{\prime}\right) \gamma^{0}\left(\boldsymbol{r}^{\prime}, \boldsymbol{r}^{\prime \prime}\right) d \boldsymbol{r}^{\prime} & =\int\left\langle\boldsymbol{r} \mid \boldsymbol{\phi}^{0}\right\rangle\left\langle\boldsymbol{\phi}^{0} \mid \boldsymbol{r}^{\prime}\right\rangle\left\langle\boldsymbol{r}^{\prime} \mid \boldsymbol{\phi}^{0}\right\rangle\left\langle\boldsymbol{\phi}^{0} \mid \boldsymbol{r}^{\prime \prime}\right\rangle d \boldsymbol{r}^{\prime} \\
& =\left\langle\boldsymbol{r} \mid \boldsymbol{\phi}^{0}\right\rangle\left\langle\boldsymbol{\phi}^{0} \mid \boldsymbol{\phi}^{0}\right\rangle\left\langle\boldsymbol{\phi}^{0} \mid \boldsymbol{r}^{\prime \prime}\right\rangle=\left\langle\boldsymbol{r} \mid \boldsymbol{\phi}^{0}\right\rangle\left\langle\boldsymbol{\phi}^{0} \mid \boldsymbol{r}^{\prime \prime}\right\rangle=\gamma^{0}\left(\boldsymbol{r}, \boldsymbol{r}^{\prime \prime}\right)
\end{aligned}
$$

Above we have used the basis set completeness, $\int d \boldsymbol{r}^{\prime}\left|\boldsymbol{r}^{\prime}\right\rangle\left\langle\boldsymbol{r}^{\prime}\right|=1$, the MO orthonormality, $\left\langle\boldsymbol{\phi}^{0} \mid \boldsymbol{\phi}^{0}\right\rangle=\mathbf{I}, \rho^{0}(\boldsymbol{r})=N p^{0}(\boldsymbol{r})$ stands for molecular electron density, while $p^{0}(\boldsymbol{r})=\gamma^{0}(\boldsymbol{r}, \boldsymbol{r}) / N$ denotes its "shape" (probability) factor.

The local information system involves these strict-localization events in both its input $\boldsymbol{a}=\{|\boldsymbol{r}\rangle\}$ and output $\boldsymbol{b}=\left\{\left|\boldsymbol{r}^{\prime}\right\rangle\right\}$. It is determined by the conditional-probability kernel

$$
P^{0}\left(\boldsymbol{r}^{\prime} \mid \boldsymbol{r}\right)=P_{\boldsymbol{r} \rightarrow \boldsymbol{r}^{\prime}}(0)=\gamma^{0}\left(\boldsymbol{r}, \boldsymbol{r}^{\prime}\right) \gamma^{0}\left(\boldsymbol{r}^{\prime}, \boldsymbol{r}\right) / \gamma^{0}(\boldsymbol{r}, \boldsymbol{r}) \equiv\left[A_{\boldsymbol{r} \rightarrow \boldsymbol{r}^{\prime}}(0)\right]^{2},
$$

the square of the associated scattering amplitude:

$$
A_{\boldsymbol{r} \rightarrow \boldsymbol{r}^{\prime}}(0)=\gamma^{0}\left(\boldsymbol{r}, \boldsymbol{r}^{\prime}\right) /\left[\rho^{0}(\boldsymbol{r})\right]^{1 / 2} .
$$

The corresponding joint-probability distribution thus reads:

$$
P^{0}\left(\boldsymbol{r}, \boldsymbol{r}^{\prime}\right)=p^{0}(\boldsymbol{r}) P^{0}\left(\boldsymbol{r}^{\prime} \mid \boldsymbol{r}\right)=\gamma^{0}\left(\boldsymbol{r}, \boldsymbol{r}^{\prime}\right) \gamma^{0}\left(\boldsymbol{r}^{\prime}, \boldsymbol{r}\right) / N
$$

One again directly verifies their normalizations using the idempotency property of the density matrix [Eq. (21)]:

$$
\begin{aligned}
\int P^{0}\left(\boldsymbol{r}^{\prime}, \boldsymbol{r}^{\prime}\right) d \boldsymbol{r}^{\prime} & =(1 / N) \int \gamma^{0}\left(\boldsymbol{r}, \boldsymbol{r}^{\prime}\right) \gamma^{0}\left(\boldsymbol{r}^{\prime}, \boldsymbol{r}\right) d \boldsymbol{r}^{\prime} \equiv(1 / N) \int \Omega^{0}\left(\boldsymbol{r}, \boldsymbol{r}^{\prime}\right) d \boldsymbol{r}^{\prime} \\
& =\gamma^{0}(\boldsymbol{r}, \boldsymbol{r}) / N=p^{0}(\boldsymbol{r}), \\
\int P^{0}\left(\boldsymbol{r}^{\prime} \mid \boldsymbol{r}\right) d \boldsymbol{r}^{\prime} & =\left[\rho^{0}(\boldsymbol{r})\right]^{-1} \int \gamma^{0}\left(\boldsymbol{r}, \boldsymbol{r}^{\prime}\right) \gamma^{0}\left(\boldsymbol{r}^{\prime}, \boldsymbol{r}\right) d \boldsymbol{r}^{\prime}=\gamma^{0}(\boldsymbol{r}, \boldsymbol{r}) / \rho^{0}(\boldsymbol{r})=1 .
\end{aligned}
$$

Therefore, the molecular density matrix uniquely determines all local communications between the system infinitesimal volume-elements, via the subspace of the configuration occupied SMO,

$$
\left\{P^{0}\left(\boldsymbol{r}, \boldsymbol{r}^{\prime}\right)=P_{\boldsymbol{r}, \boldsymbol{r}^{\prime}}\left[\gamma^{0}\right]\right\}, \quad\left\{P^{0}\left(\boldsymbol{r}^{\prime} \mid \boldsymbol{r}\right)=P_{\boldsymbol{r} \rightarrow \boldsymbol{r}^{\prime}}\left[\gamma^{0}\right]\right\} \quad \text { and } \quad\left\{A^{0}\left(\boldsymbol{r}^{\prime} \mid \boldsymbol{r}\right)=A_{\boldsymbol{r} \rightarrow \boldsymbol{r}^{\prime}}\left[\gamma^{0}\right]\right\}
$$

While probabilities of the local conditional probabilities are independent of the phases of the "off-diagonal" part of $\gamma^{0}$, for $\boldsymbol{r}$ ' $\neq \boldsymbol{r}$, their amplitudes are seen to be explicitly dependent upon phases of the local basis functions. 


\section{Entropy/information descriptors of local channels}

The overall conditional entropy descriptor [Eq. (3)] of the local channel defined by the $t w o$-point probabilities of Eqs. (22) and (24), is given by the following functional of the density matrix $\gamma^{0}$ of the molecular ground-state configuration $\Psi_{0}(N)$ [see Eq. (21)],

$$
\begin{aligned}
S^{0}\left(\Re^{\prime} \mid \Re\right)= & -\iint P^{0}\left(\boldsymbol{r}, \boldsymbol{r}^{\prime}\right) \log P^{0}\left(\boldsymbol{r}^{\prime} \mid \boldsymbol{r}\right) d \boldsymbol{r} d \boldsymbol{r}^{\prime} \\
= & -\iint P_{\boldsymbol{r}, \boldsymbol{r}^{\prime}}\left[\gamma^{0}\right] \log P_{\boldsymbol{r} \rightarrow \boldsymbol{r}^{\prime}}\left[\gamma^{0}\right] d \boldsymbol{r} d \boldsymbol{r}^{\prime} \equiv S_{\mathfrak{R} \rightarrow \Re^{\prime}}\left[\gamma^{0}\right] \\
= & -N^{-1} \iint \gamma^{0}\left(\boldsymbol{r}, \boldsymbol{r}^{\prime}\right) \gamma^{0}\left(\boldsymbol{r}^{\prime}, \boldsymbol{r}\right)\left\{\log \left[\gamma^{0}\left(\boldsymbol{r}, \boldsymbol{r}^{\prime}\right) \gamma^{0}\left(\boldsymbol{r}, \boldsymbol{r}^{\prime}\right)\right]-\log \gamma^{0}(\boldsymbol{r}, \boldsymbol{r})\right\} d \boldsymbol{r}^{\prime} d \boldsymbol{r} \\
\equiv & -N^{-1} \iint \Omega^{0}\left(\boldsymbol{r}, \boldsymbol{r}^{\prime}\right) \log \Omega^{0}\left(\boldsymbol{r}, \boldsymbol{r}^{\prime}\right) d \boldsymbol{r}^{\prime} d \boldsymbol{r} \\
& +N^{-1} \int\left[\int \Omega^{0}\left(\boldsymbol{r}, \boldsymbol{r}^{\prime}\right) d \boldsymbol{r}^{\prime}\right] \log \rho^{0}(\boldsymbol{r}) d \boldsymbol{r} \\
= & N^{-1}\left(S\left[\Omega^{0}\right]-S\left[\rho^{0}\right]\right) .
\end{aligned}
$$

It thus reflects the difference between the Shannon-entropy

$$
S\left[\Omega^{0}\right]=-\iint \Omega^{0}\left(\boldsymbol{r}, \boldsymbol{r}^{\prime}\right) \log \Omega^{0}\left(\boldsymbol{r}, \boldsymbol{r}^{\prime}\right) d \boldsymbol{r} d \boldsymbol{r}^{\prime}
$$

of the two-point distribution of Eq. (25),

$$
\Omega^{0}\left(\boldsymbol{r}, \boldsymbol{r}^{\prime}\right)=\Omega_{\boldsymbol{r}, \boldsymbol{r}^{\prime}}^{0}\left[\gamma^{0}\right]=\gamma^{0}\left(\boldsymbol{r}, \boldsymbol{r}^{\prime}\right) \gamma^{0}\left(\boldsymbol{r}^{\prime}, \boldsymbol{r}\right)=N P^{0}\left(\boldsymbol{r}, \boldsymbol{r}^{\prime}\right)
$$

and the entropy of the electron density $\rho^{0}(\boldsymbol{r})=N p^{0}(\boldsymbol{r})$,

$$
\begin{aligned}
S\left[\rho^{0}\right] & =-\int \rho^{0}(\boldsymbol{r}) \log \rho^{0}(\boldsymbol{r}) d \boldsymbol{r}=-N \int p^{0}(\boldsymbol{r})\left[\log N+\log p^{0}(\boldsymbol{r})\right] d \boldsymbol{r} \\
& =-N \log N+N S\left[p^{0}\right] .
\end{aligned}
$$

The deterministic contribution $S^{0}$ (diag.) of $S\left[\Omega^{0}\right]$ originates from its "diagonal" scatterings $\{\boldsymbol{r} \rightarrow \boldsymbol{r}\}$, when $\Omega^{0}(\boldsymbol{r}, \boldsymbol{r})=\left[\rho^{0}(\boldsymbol{r})\right]^{2}$ and hence:

$$
\begin{aligned}
S^{\text {diag }} \cdot\left[\Omega^{0}\right] & =-\int \Omega^{0}(\boldsymbol{r}, \boldsymbol{r}) \log \Omega^{0}(\boldsymbol{r}, \boldsymbol{r}) d \boldsymbol{r}=-\int\left[\rho^{0}(\boldsymbol{r})\right]^{2} \log \left[\rho^{0}(\boldsymbol{r})\right]^{2} d \boldsymbol{r}=S\left[\left(\rho^{0}\right)^{2}\right] \\
& =-2 N^{2} \int\left[p^{0}(\boldsymbol{r})\right]^{2} \log \left[N p^{0}(\boldsymbol{r})\right] d \boldsymbol{r},
\end{aligned}
$$

i.e., by the diagonal contribution to $S_{\mathfrak{R} \rightarrow \mathfrak{R}^{\prime}}\left[\gamma^{0}\right]$ :

$$
S^{0}(\text { diag. })=N^{-1}\left\{S^{\text {diag. }}\left[\Omega^{0}\right]-S\left[\rho^{0}\right]\right\}=N^{-1}\left\{S\left[\left(\rho^{0}\right)^{2}\right]-S\left[\rho^{0}\right]\right\} .
$$

Accordingly, the "scattering" aspect of $S\left[\Omega^{0}\right]$ is revealed by its off-diagonal part

$$
S^{\text {ndiag. }} \cdot\left[\Omega^{0}\right]=S\left[\Omega^{0}\right]-S^{\text {diag. }} \cdot\left[\Omega^{0}\right],
$$

or by the associated contribution to $S_{\mathfrak{R} \rightarrow \Re^{\prime}}\left[\gamma^{0}\right]$ :

$$
S^{0}(\text { ndiag. })=S^{0}\left(\Re^{\prime} \mid \Re\right)-S^{0}(\text { diag. })=N^{-1} S^{\text {ndiag. }} \cdot\left[\Omega^{0}\right] \text {. }
$$


The electron distribution of the system promolecule $[11,35]$ is described by the sum of the molecularly placed electron densities $\left\{\rho_{0}^{\mathrm{X}}(\boldsymbol{r})\right\}$ of the free constituent atoms,

$$
\rho_{0}(\boldsymbol{r})=N p_{0}(\boldsymbol{r})=\sum_{\mathrm{X}} \rho_{\mathrm{X}}^{0}(\boldsymbol{r})
$$

determining the diagonal part $\rho_{0}(\boldsymbol{r})=\gamma_{0}(\boldsymbol{r}, \boldsymbol{r})$ of the promolecular density matrix

$$
\gamma_{0}\left(\boldsymbol{r}, \boldsymbol{r}^{\prime}\right)=\sum_{\mathrm{X}} \gamma_{\mathrm{X}}^{0}(\boldsymbol{r}, \boldsymbol{r})
$$

The shape factor $p_{0}(\boldsymbol{r})$ provides the reference input signal for establishing the corresponding descriptor of the information-flow in the local channel, given by the mutual information quantity of Eqs. (7) and (9):

$$
\begin{aligned}
I^{0}\left(\Re_{0}: \Re^{\prime}\right) & =N^{-1} \int\left[\int P^{0}\left(\boldsymbol{r}, \boldsymbol{r}^{\prime}\right)\right] \log \left[P^{0}\left(\boldsymbol{r}^{\prime} \mid \boldsymbol{r}\right) / p_{0}(\boldsymbol{r})\right] d \boldsymbol{r}^{\prime} d \boldsymbol{r} \equiv I_{\Re \rightarrow \Re^{\prime}}^{0}\left[\gamma^{0}, \gamma_{0}\right] \\
& \left.=-S_{\Re \rightarrow \Re^{\prime}}\left[\gamma^{0}\right]-N^{-1} \int\left[\int \Omega^{0}\left(\boldsymbol{r}, \boldsymbol{r}^{\prime}\right) d \boldsymbol{r}^{\prime}\right] \log p_{0}(\boldsymbol{r})\right\} d \boldsymbol{r} \\
& \equiv-S_{\mathfrak{R} \rightarrow \mathfrak{R}^{\prime}}\left[\gamma^{0}\right]+S\left[p^{0}\right]+\Delta S\left[p^{0} \mid p_{0}\right]
\end{aligned}
$$

where the entropy deficiency in the molecular (ground-state) probability $p^{0}$ relative to the promolecular distribution $p_{0}$ [compare Eq. (8)],

$$
\Delta S\left[p^{0} \mid p_{0}\right]=\int p^{0}(\boldsymbol{r}) \log \left[p^{0}(\boldsymbol{r}) / p_{0}(\boldsymbol{r})\right] d \boldsymbol{r} \equiv \int p^{0}(\boldsymbol{r}) I(\boldsymbol{r}) d \boldsymbol{r}
$$

measures the average value of the local surprisal function $I(\boldsymbol{r})$, which reflects the information distance between the two distributions. It generally constitutes a relatively minor contribution, since $p^{0}(\boldsymbol{r})$ and $p_{0}(\boldsymbol{r})$ strongly resemble one another, being distinguished only by relatively minor displacements in the AIM valence shells. This similarity index exactly disappears for the molecular input signal $p^{0}(\boldsymbol{r}), \Delta S\left[p^{0}\right.$ $\left.\mid p^{0}\right]=0$, thus giving a modified value of the average mutual information of the local channel [Eq. (10)]:

$$
I^{0}\left(\Re: \Re^{\prime}\right)=-S_{\mathfrak{i} \rightarrow \Re^{\prime}}\left[\gamma^{0}\right]+S\left[p^{0}\right] .
$$

The corresponding overall bond multiplicity indices of Eqs. (11) and (12) then read:

$$
\begin{aligned}
& M^{0}\left(\Re_{0} ; \Re^{\prime}\right)=S^{0}\left(\Re^{\prime} \mid \Re\right)+I^{0}\left(\Re_{0} ; \Re^{\prime}\right)=S\left[p^{0}\right]+\Delta S\left[p^{0} \mid p_{0}\right], \\
& M^{0}\left(\Re ; \Re^{\prime}\right)=S^{0}\left(\Re^{\prime} \mid \Re\right)+I^{0}\left(\Re ; \Re^{\prime}\right)=S\left[p^{0}\right] .
\end{aligned}
$$

Therefore, the overall IT multiplicity of the molecular local channel probed by the molecular signal again recovers the Shannon entropy of the ground-state probability of electrons [compare Eq. (12)]. The difference index of Eq. (13),

$$
\Delta M=M^{0}\left(\Re ; \Re^{\prime}\right)-M^{0}\left(\Re_{0} ; \Re^{\prime}\right)=\Delta S\left[p^{0} \mid p_{0}\right],
$$


again reflects the overall similarity between the molecular probability distribution $p^{0}$ and the promolecular reference $p_{0}$; it correctly disappears in the Separated Atoms Limit (SAL), where the two distributions become identical.

\section{Average orbital communications in CI MO-ensembles}

Consider next the familiar (single-reference) Configuration Interaction (CI) expansion of the molecular ground-state $\Psi^{\mathrm{CI}}(N)$ into $n_{c}$ Slater determinants (electron configurations) $\boldsymbol{\Psi}(N)=\left\{\Psi_{\alpha}(N)\right\}$ :

$$
\begin{aligned}
\Psi^{\mathrm{CI}}(N)= & \sum_{\alpha} \Psi_{\alpha}(N) c_{\alpha} \equiv \boldsymbol{\Psi}(N) \boldsymbol{c}, \\
\boldsymbol{c}= & \left\{c_{\alpha}=\left\langle\Psi_{\alpha}(N) \mid \Psi^{\mathrm{CI}}(N)\right\rangle, \alpha=0,1, \ldots, n_{c}\right\}=\left\langle\boldsymbol{\Psi}(N) \mid \Psi^{\mathrm{CI}}(N)\right\rangle, \\
& \left\langle\Psi_{\alpha}(N) \mid \Psi_{\beta}(N)\right\rangle=\delta_{\alpha, \beta} \text { or }\langle\boldsymbol{\Psi}(N) \mid \boldsymbol{\Psi}(N)\rangle=\mathbf{I},
\end{aligned}
$$

with $\alpha=0$ corresponding to the ground-state (HF or KS) configuration $\Psi_{0}(N)$, which usually dominates. The single-configuration approximation of Eq. (14), for $\left|c_{0}\right|=1$ or $p_{0}=\left|c_{0}\right|^{2}=1$, can be thus regarded as the limiting case of the CI expansion truncated after this first $\mathrm{HF} / \mathrm{KS}$ configuration. A typical strong dominance of the CI wave function by the HF/KS ground-state configuration, $p_{\alpha=0}>>p_{\alpha>0}$, implies that most of the AO communications are indeed carried out via the SMO occupied in the $\mathrm{HF} / \mathrm{KS}$ Slater determinant, which generally provides quite satisfactory description of gross patterns of chemical bonds, e.g., [11-21].

The (orthonormal) configurations $\boldsymbol{\Psi}(N)=\left\{\Psi_{\alpha}(N)=\operatorname{det}\left[\varphi^{\alpha}\right]\right\}$ are uniquely determined by the subspaces of $N$ singly-occupied SMO, $\varphi^{\alpha}$, or by their AO expansions, $\varphi^{\alpha}=\chi \boldsymbol{C}^{\alpha}=\left\{\varphi_{s}(\alpha)=\chi \boldsymbol{C}_{s}^{\alpha}\right\}, \mathbf{C}^{\alpha}=\left\{C_{i, s}(\alpha)=\left\langle\chi_{i} \mid \varphi_{s}(\alpha)\right\rangle\right\}$, which give rise to the associated $\mathrm{CBO}$ matrices [see Eq. (16)],

$$
\boldsymbol{\gamma}^{\alpha}=\left\langle\chi \mid \varphi^{\alpha}\right\rangle\left\langle\varphi^{\alpha} \mid \chi\right\rangle=\mathbf{C}^{\alpha} \mathbf{C}^{\alpha \dagger}=\mathbf{C v}^{\alpha} \mathbf{C}^{\dagger}
$$

Here, the (diagonal) matrix of SMO-occupations in the configuration $\Psi_{\alpha}(N)$,

$$
v^{\alpha}=\left\{[1 \text { (occupied } \mathrm{MO}) \text { or } 0(\text { virtual MO) }] \delta_{s, s^{\prime}}=v_{s}(\alpha) \delta_{s, s^{\prime}}\right\}
$$

and the full LCAO matrix $\mathbf{C}=\left(\mathbf{C}_{i}^{\alpha} \mathbf{C}^{v, \alpha}\right)$ combines expansion coefficients of all $m$ MO:

$$
\boldsymbol{\varphi}=\chi \mathbf{C}=\left(\varphi^{\alpha}, \varphi^{v, \alpha}\right)=\left\{\varphi_{s}\right\}
$$

occupied, $\varphi^{\alpha}$, and virtual, $\varphi^{v, \alpha}=\chi \mathbf{C}^{v, \alpha}$, obtained in the given basis set $\chi=$ $\left(\chi_{1}, \chi_{2}, \ldots, \chi_{m}\right)$. The diagonal elements of $\gamma^{\alpha}$ represent the configuration effective AO occupations $\left\{n_{i}(\alpha)=\gamma_{i, i}(\alpha)\right\}$, while the off-diagonal elements similarly determine the configuration "bond-orders" between different AO: $\left\{n_{i, j}(\alpha)=\gamma_{i, j}(\alpha)\right.$, $i \neq j\}$. 
Therefore, each configuration can be associated with the related MO-ensemble defined by the idempotent SMO-projector $\hat{\mathrm{P}}_{\alpha}=\left|\varphi^{\alpha}\right\rangle\left\langle\varphi^{\alpha}\right|$ :

$$
\begin{aligned}
\hat{\mathrm{d}}_{\alpha} & =\sum_{s}\left[v_{s}(\alpha) / N\right]\left|\phi_{s}\right\rangle\left\langle\phi_{s}\left|\equiv \sum_{s} \pi_{s}(\alpha) \hat{\mathrm{P}}_{s}=(1 / N)\right| \varphi^{\alpha}\right\rangle\left\langle\varphi^{\alpha}\right| \\
& =(1 / N) \hat{\mathrm{P}}_{\alpha} ; \quad \sum_{s} \pi_{s}(\alpha)=1 .
\end{aligned}
$$

In other words, each determinant $\Psi_{\alpha}(N)$ implies the associated ensemble of the configuration occupied SMO (single-particle states). It is defined by the density operator $\hat{\mathrm{d}}_{\alpha}$ with equal probabilities of the occupied (physical) SMO in $\Psi_{\alpha}(N),\left\{\pi_{s \in \alpha}=1 / N\right\}$, through which the electron probabilities are scattered (delocalized) between $m$ basis functions in the underlying AO information system. One further observes that the CBO (density) matrix $\boldsymbol{\gamma}^{\alpha}$ is proportional to the $\mathrm{AO}$ representation of $\hat{\mathrm{d}}_{\alpha}$,

$$
\mathbf{d}^{\alpha} \equiv\left\langle\chi\left|\hat{\mathrm{d}}_{\alpha}\right| \chi\right\rangle=N^{-1} \gamma^{\alpha}
$$

The combination of configurations in the ground state $\Psi^{\mathrm{CI}}(N)$ [Eq. (43)] similarly determines the $\mathrm{CI} \mathrm{MO}$-ensemble corresponding to non-equal SMO probabilities, $\left\{\pi_{s}=\sum_{\alpha} p_{\alpha} \pi_{s}(\alpha)\right\}$

$$
\hat{\mathrm{d}}^{\mathrm{CI}}=\sum_{s=1}^{m} \pi_{s} \hat{\mathrm{P}}_{s}, \quad \sum_{s} \pi_{s}=1,
$$

where the conditional probability $p_{\alpha}$ of the configuration $\Psi_{\alpha}(N)$ in $\Psi^{\mathrm{CI}}(N)$ results from the superposition principle:

$$
p_{\alpha} \equiv P\left(\Psi_{\alpha} \mid \Psi^{\mathrm{CI}}\right)=\left|c_{\alpha}\right|^{2}, \quad \sum_{\alpha} p_{\alpha}=1
$$

This CI density operator of MO subsequently determines its AO representation,

$$
\mathbf{d}^{\mathrm{CI}} \equiv\left\langle\chi\left|\hat{\mathrm{d}}^{\mathrm{CI}}\right| \chi\right\rangle \equiv N^{-1} \boldsymbol{\gamma}^{\mathrm{CI}}
$$

proportional to the CI-average density matrix matrix:

$$
\boldsymbol{\gamma}^{\mathrm{CI}}=\sum_{\alpha} p_{\alpha} \boldsymbol{\gamma}^{\alpha} \equiv\langle\boldsymbol{\gamma}\rangle_{\text {ens. }} .
$$

The latter combines the diagonal elements $\left\{\gamma_{i, i}(\alpha)=n_{i}(\alpha)\right\}$, the average AO occupations in the configurations involved in the CI ensemble defined by the density operator

$$
\hat{\mathrm{D}}^{\mathrm{CI}}=\sum_{\alpha}\left|\Psi_{\alpha}\right\rangle p_{\alpha}\left\langle\Psi_{\alpha}\right|=\sum_{\alpha} p_{\alpha} \hat{\mathrm{P}}_{\alpha},
$$

defined by sum of the configuration projections $\left\{\hat{\mathrm{P}}_{\alpha}\right\}$ weighted by the associated probabilities $\boldsymbol{p}^{\text {ens. }}=\left\{p_{\alpha}\right\}$, 


$$
n_{i}^{\mathrm{CI}}=\left\langle n_{i}\right\rangle_{\text {ens. }}=\sum_{\alpha} p_{\alpha} n_{i}(\alpha) \equiv\left\langle\gamma_{i, i}\right\rangle_{\text {ens. }}=\sum_{\alpha} p_{\alpha} \gamma_{i, i}(\alpha), \quad i=1,2, \ldots,
$$

and the off-diagonal elements reflecting the CI-average "bond-orders" between pairs of basis functions:

$$
n_{i, j}^{\mathrm{CI}}=\left\langle n_{i, j}\right\rangle_{\text {ens. }}=\sum_{\alpha} p_{\alpha} n_{i, j}(\alpha)=\sum_{\alpha} p_{\alpha} \gamma_{i, j}(\alpha)=\left\langle\gamma_{i, j}\right\rangle_{\text {ens. }}, \quad i \neq j .
$$

In principle there are two admissible ways of extending the molecular $\mathrm{AO}$ communications into the above CI scenario involving several electronic configurations: the classical (probability) and quantum (amplitude) averaging in the ground-state CI SMO-ensemble. Both ultimately reproduce the single-configuration development of the preceding section as their limiting case, but only the latter is capable of accounting for the interference phenomena between AO communications in the configurations used in the CI expansion of the system ground state. Evaluating capabilities of these two treatments in interpreting the prototype bond patterns should in principle allow one to select the most suitable approach for chemical applications, to be used for an understanding of the information content of molecular electronic structure, patterns of chemical bonds, bond multiplicities and their covalent/ionic composition.

The classical (probability) CI channel thus involves the averaging over the configuration $\mathrm{AO}$ probabilities themselves:

$$
\begin{aligned}
\langle P(j \mid i)\rangle_{\text {ens. }} & \equiv \sum_{\alpha} p_{\alpha} P^{\alpha}(j \mid i) \equiv \sum_{\alpha} p_{\alpha} P_{i \rightarrow j}(\alpha) \\
& =\left\langle N_{i}\right\rangle_{\text {ens. }} \sum_{\alpha} p_{\alpha} \gamma_{i, j}(\alpha) \gamma_{j, i}(\alpha), \\
\sum_{j}\langle P(j \mid i)\rangle_{\text {ens. }} & =1 \Rightarrow\left\langle N_{i}\right\rangle_{\text {ens. }}=1 /\left\langle n_{i}\right\rangle_{\text {ens. }} .
\end{aligned}
$$

Here the elements of the AO conditional-probability matrix in configuration $\Psi_{\alpha}(N)$ [see Eq. (17)],

$$
\begin{aligned}
\mathbf{P}^{\alpha}\left(\boldsymbol{\chi}^{\prime} \mid \boldsymbol{\chi}\right)= & \left\{P^{\alpha}(j \mid i) \equiv P_{i \rightarrow j}(\alpha)\right. \\
= & \left.\left|A_{i \rightarrow j}(\alpha)\right|^{2}=\left|\gamma_{i, j}(\alpha)\right|^{2} / \gamma_{i, i}(\alpha) \equiv N_{i}(\alpha) \gamma_{i, j}(\alpha)^{2}\right\}, \\
& \alpha=0,1, \ldots, n_{c}
\end{aligned}
$$

determine the configuration a posteriori (classical) amplitudes [see Eq. (19)]:

$$
\begin{aligned}
\mathbf{A}^{\alpha}\left(\chi^{\prime} \mid \chi\right) & =\left\{A^{\alpha}(j \mid i) \equiv A_{i \rightarrow j}(\alpha) \equiv\left[P^{\alpha}(j \mid i)\right]^{1 / 2}=\gamma_{i, j}(\alpha) /\left[\gamma_{i, i}(\alpha)\right]^{1 / 2}\right. \\
& \left.=\left[N_{i}(\alpha)\right]^{1 / 2} \gamma_{i, j}(\alpha)\right\} .
\end{aligned}
$$

The average probabilities $\left\langle\mathbf{P}\left(\chi^{\prime} \mid \chi\right)\right\rangle_{\text {ens. }}=\left\{\langle P(j \mid i)\rangle_{\text {ens. }} \equiv\left\langle P_{i \rightarrow j}\right\rangle_{\text {ens. }}\right\}$ generate the classical (probability) network of the "single-input" summation (parallel) CI arrangement [85]: 


$$
\begin{aligned}
& \chi \rightarrow\left\{p_{\alpha} \rightarrow \mathbf{P}^{\alpha}\left(\chi^{\prime} \mid \chi\right)\right\} \rightarrow \chi^{\prime} \Rightarrow \chi \rightarrow\left\langle\mathbf{P}\left(\chi^{\prime} \mid \chi\right)\right\rangle_{\text {ens. }} \rightarrow \chi^{\prime} \\
& \left\langle\mathbf{P}\left(\chi^{\prime} \mid \chi\right)\right\rangle_{\text {ens. }}=\sum_{\alpha} p_{\alpha} \mathbf{P}^{\alpha}\left(\chi^{\prime} \mid \chi\right) \equiv\left\{\left[\left\langle A_{i \rightarrow j}\right\rangle_{\text {ens. }}\right]^{2}\right\}
\end{aligned}
$$

where $\left\langle A_{i \rightarrow j}\right\rangle_{\text {ens. }}$. denotes the a posteriori $\mathrm{CI}$ amplitude for the $\chi_{i} \rightarrow \chi_{j}$ communication. In this approximation the ensemble-average quantity $\langle P(j \mid i)\rangle_{\text {ens. }}$. describes the classical $\chi_{i} \rightarrow \chi_{j}$ AO probability propagation in the ground-state CI combination of configurations. It misses the amplitude-superposition terms, which reflect quantum effects of the direct $\mathrm{AO}$ communications (bonds) in a molecule. This direct $\mathrm{CI}$ averaging of the configuration $\mathrm{AO}$ communications also involves an effective MO-ensemble defined by the (non-idempotent) density operator of Eq. (49),

$$
\hat{\mathrm{d}}^{\mathrm{CI}}=\sum_{\alpha} p_{\alpha} \hat{\mathrm{d}}_{\alpha}=\sum_{s}\left\{\sum_{\alpha} p_{\alpha}\left[v_{s}(\alpha) / N\right]\right\}\left|\phi_{s}\right\rangle\left\langle\phi_{s}\right| \equiv \sum_{s} \pi_{s} \hat{\mathrm{P}}_{s}, \quad \sum_{s} \pi_{s}=1,
$$

exhibiting non-equal probabilities of SMO, $\left\{\pi_{s}=v_{s}^{\mathrm{CI}} / N\right\}$, which reflect the effective (fractional) average occupations $\left\{v_{s}^{\mathrm{CI}}=\sum_{\alpha} p_{\alpha} v_{s}(\alpha)\right\}$ of SMO in the CI-ensemble. As also indicated in Eq. (59) the square root of each probability $\langle P(j \mid i)\rangle_{\text {ens. }}$ determines the corresponding classical CI amplitude $\langle A(j \mid i)\rangle_{\text {ens. }}=\left[\langle P(j \mid i)\rangle_{\text {ens. }}\right]^{1 / 2}$.

In the quantum, amplitude-averaging scheme the elements of $\langle\boldsymbol{\gamma}\rangle_{\text {ens. }}$. are first used to determine the resultant (a priori) amplitudes $\left\{\left\langle A_{i \rightarrow j}\right\rangle_{a v}\right\}$ of the average communications between AO in this CI-ensemble of SMO. In full analogy to Eq. (19) this resultant communication amplitude is shaped by the ensemble average of the corresponding $\mathrm{CBO}$ element,

$$
\begin{aligned}
\langle A(j \mid i)\rangle_{a v .} & \equiv\left\langle A_{i \rightarrow j}\right\rangle_{a v .}=\left[\left\langle N_{i}\right\rangle_{a v .}\right]^{1 / 2}\left\langle\gamma_{i, j}\right\rangle_{e n s .}=\left[\left\langle N_{i}\right\rangle_{a v .}\right]^{1 / 2} \sum_{\alpha} p_{\alpha} \gamma_{i, j}(\alpha) \quad \text { or } \\
\left\langle\mathbf{A}\left(\boldsymbol{\chi}^{\prime} \mid \chi\right)\right\rangle_{a v .} & =\sum_{\alpha} p_{\alpha} \mathbf{A}^{\alpha}\left(\boldsymbol{\chi}^{\prime} \mid \chi\right)=\left\{\langle A(j \mid i)\rangle_{a v .} \equiv\left\langle A_{i \rightarrow j}\right\rangle_{a v .}\right\}
\end{aligned}
$$

with $\left\langle N_{i}\right\rangle_{a v}$. standing for the normalization factor of the resultant (average) conditional probabilities

$$
\begin{aligned}
\langle P(j \mid i)\rangle_{a v} . & \equiv\left|\left\langle A_{i \rightarrow j}\right\rangle_{a v}\right|^{2}=\left\langle N_{i}\right\rangle_{a v} .\left\langle\gamma_{i, j}\right\rangle_{\text {ens. }}\left\langle\gamma_{j, i}\right\rangle_{\text {ens } .} . \\
& =\left\langle N_{i}\right\rangle_{a v} . \sum_{\alpha} \sum_{\beta} p_{\alpha} p_{\beta} \gamma_{i, j}(\alpha) \gamma_{j, i}(\beta) \\
& \equiv \sum_{\alpha} \sum_{\beta} p_{\alpha} p_{\beta} P_{i \rightarrow j}(\alpha, \beta),
\end{aligned}
$$

now containing terms responsible for the interference between configurations. The normalization constant $\left\langle N_{i}\right\rangle_{a v}$. is again obtained from the requirement $\sum_{j}\langle P(j \mid i)\rangle_{a v}$. $=1$ : 


$$
\left\langle N_{i}\right\rangle_{a v .}=\left\{\sum_{\alpha} \sum_{\beta} p_{\alpha} p_{\beta}\left[\sum_{j} \gamma_{i, j}(\alpha) \gamma_{j, i}(\beta)\right]\right\}^{-1} .
$$

For the complete basis, when $\sum_{j}\left|\chi_{j}\right\rangle\left\langle\chi_{j}\right|=1$, it reads:

$$
\left\langle N_{i}\right\rangle_{a v .}=\left\{\left\langle\chi_{i}\left|\left[\sum_{s}\left|\varphi_{s}\right\rangle\left(v_{s}^{\mathrm{CI}}\right)^{2}\left\langle\varphi_{s}\right|\right]\right| \chi_{i}\right\rangle\right\}^{-1} .
$$

The resultant amplitudes of Eq. (61) define the quantum (amplitude) channel in the CI ground-state,

$$
|\chi\rangle \rightarrow\left\{p_{\alpha} \rightarrow \mathbf{A}^{\alpha}\left(\chi^{\prime} \mid \chi\right)\right\} \rightarrow\left|\chi^{\prime}\right\rangle \Rightarrow|\chi\rangle \rightarrow\left\langle\mathbf{A}\left(\chi^{\prime} \mid \chi\right)\right\rangle_{a v .} \rightarrow\left|\chi^{\prime}\right\rangle
$$

while the average probabilities of Eq. (62), $\left\langle\mathbf{P}\left(\boldsymbol{\chi}^{\prime} \mid \boldsymbol{\chi}\right)\right\rangle_{a v} .=\left\{\left\langle P_{i \rightarrow j}\right\rangle_{a v}\right.$. $\}$, the squares of these average amplitudes, generate the associated quantum probability network. The latter is seen to be determined by the following "double-input" (parallel) system of the amplitude propagations in the CI MO-ensemble,

$$
\begin{aligned}
& |\chi\rangle \rightarrow\left\{p_{\alpha} \rightarrow \mathbf{A}^{\alpha}\left(\chi^{\prime} \mid \chi\right)\right\} \rightarrow\left|\chi^{\prime}\right\rangle \leftarrow\left\{\mathbf{A}^{\beta}\left(\chi^{\prime} \mid \chi^{\prime \prime}\right) \leftarrow p_{\beta}\right\} \leftarrow\left|\chi^{\prime \prime}\right\rangle \\
& \quad \Rightarrow|\chi\rangle \rightarrow\left\langle\mathbf{A}\left(\chi^{\prime} \mid \chi\right)\right\rangle_{a v .} \rightarrow\left|\chi^{\prime}\right\rangle \leftarrow\left\langle\mathbf{A}\left(\chi^{\prime} \mid \chi^{\prime \prime}\right)\right\rangle_{a v} \leftarrow\left|\chi^{\prime \prime}\right\rangle .
\end{aligned}
$$

In short notation, for $\chi=\chi^{\prime \prime}$, it gives rise to the resultant probability propagation of Eq. (62):

$$
\chi \rightarrow\left\langle\mathbf{P}\left(\chi^{\prime} \mid \chi\right)\right\rangle_{a v} \rightarrow \chi^{\prime}
$$

Therefore, the average probability $\langle P(j \mid i)\rangle_{a v}$. describes the parallel, double-input scatterings via the average propagation amplitudes:

$$
\begin{aligned}
& \left|\chi_{i}\right\rangle \rightarrow\left\{p_{\alpha} \rightarrow A_{i \rightarrow j}(\alpha)\right\} \rightarrow\left|\chi_{j}\right\rangle \leftarrow\left\{A_{j \leftarrow i}(\beta) \leftarrow p_{\beta}\right\} \leftarrow\left|\chi_{i}\right\rangle \\
& \quad \Rightarrow\left|\chi_{i}\right\rangle \rightarrow\left\langle A_{i \rightarrow j}\right\rangle_{a v} \rightarrow\left|\chi_{j}\right\rangle \leftarrow\left\langle A_{i \rightarrow j}\right\rangle_{a v} \leftarrow\left|\chi_{i}\right\rangle \text { or } \chi_{i} \rightarrow\left\langle P_{i \rightarrow j}\right\rangle_{a v} \rightarrow \chi_{j} .
\end{aligned}
$$

The average probability $\left\langle P_{i \rightarrow j}\right\rangle_{a v}$. thus represents the resultant effect of the double amplitude propagations $\left|\chi_{i}\right\rangle \rightarrow\left|\chi_{j}\right\rangle \leftarrow\left|\chi_{i}\right\rangle$ and differs, by communication contributions reflecting the inter-configuration-interference, from the direct ensemble-average $\left\langle P_{i \rightarrow j}\right\rangle_{e n s}$. of the configuration conditional probabilities.

To summarize, the classical interpretation of molecular conditional probabilities, defining the ensemble-average $\mathrm{AO}$ communications of the $\mathrm{CI}$ configurations, is phase-independent since both the ensemble weights $\left\{p_{\alpha}\right\}$ and the configuration probabilities $\left\{\mathbf{P}^{\alpha}\left(\boldsymbol{\chi}^{\prime} \mid \boldsymbol{\chi}\right)\right\}$ loose memory about the phase content of both the CI coefficients $\left\{c_{\alpha}\right\}$ and the CBO matrix elements. In order to predict the interference effects between communication amplitudes of configurations in the resultant AO probabilities, one first combines the relevant elementary amplitudes due to 
each determinant into $\left\langle\mathbf{A}\left(\boldsymbol{\chi}^{\prime} \mid \boldsymbol{\chi}\right)\right\rangle_{a v} .=\left\{\left\langle A_{i \rightarrow j}\right\rangle_{a v}\right.$. $\}$ before determining the CI average communication probabilities as the squared moduli of the resultant amplitudes, $\left\langle\mathbf{P}\left(\chi^{\prime} \mid \chi\right)\right\rangle_{a v} .=\left\{\langle P(j \mid i)\rangle_{a v} . \equiv\left|\left\langle A_{i \rightarrow j}\right\rangle_{e n s .}\right|^{2}\right\}$. As we have argued above, this probability channel can be related to the double-input amplitude propagations between AO.

The classical (probability) channel in the CI SMO-ensemble involves only $n_{c}$ elementary (intra-configuration) probability communications $\left\{P_{i \rightarrow j}(\alpha)\right\}$, while the quantum (amplitude) network explores both the intra- and inter-configuration propagations between basis functions, and exhibits an explicit phase dependence. The latter requires $n_{c}^{2}$ elementary probability networks $\left\{P_{i \rightarrow j}(\alpha, \beta)\right\}$ which imply more indeterminacy in molecular communications between AO compared to the classical channel. Therefore, the quantum treatment of the orbital communications is expected to generate an increased fraction of the "noise" (electron AO delocalization, IT-covalency) content, and hence a decreased level of the communication "determinicity" (electron AO localization, IT-ionicity), compared to those characterizing the classical channel.

\section{Illustrative example: $t w o$-orbital model of chemical bond}

As an illustrative case consider the simplest 2-AO model consisting of the two orthonormal basis functions, e.g., two symmetrically (Löwdin) orthogonalized (real) AO contributed by different atoms $\mathrm{A}$ and $\mathrm{B}: \chi=\chi^{\prime}=\left(\chi_{1}, \chi_{2}\right) \equiv\left(\chi_{\mathrm{A}}, \chi_{\mathrm{B}}\right)$. They give rise to two independent (spatial) MO combinations $\phi=\left(\phi_{b}, \phi_{a}\right)$, which can be expressed in terms of the complementary AO probabilities $P$ and $Q=1-P$,

bonding, $\phi_{b}=\chi_{\mathrm{A}} C_{\mathrm{A}, b}+\chi_{\mathrm{B}} C_{\mathrm{B}, b} \equiv \chi_{\mathrm{A}}(P)^{1 / 2}+\chi_{\mathrm{B}}(Q)^{1 / 2} \equiv \chi C_{b}$, and anti-bonding, $\phi_{a}^{(1)}=\chi_{\mathrm{A}} C_{\mathrm{A}, a}^{(1)}+\chi_{\mathrm{B}} C_{\mathrm{B}, a}^{(1)} \equiv-\chi_{\mathrm{A}}(Q)^{1 / 2}+\chi_{\mathrm{B}}(P)^{1 / 2} \equiv \chi C_{a}^{(1)}$,

or in the compact joint notation:

$$
\boldsymbol{\phi}^{(1)}=\chi\left[\boldsymbol{C}_{b^{\prime}} \boldsymbol{C}_{a}^{(1)}\right] \equiv \chi \mathbf{C}^{(1)}, \quad \mathbf{C}^{(1)}=\left[\begin{array}{cc}
\sqrt{P} & -\sqrt{Q} \\
\sqrt{Q} & \sqrt{P}
\end{array}\right],
$$

The magnitudes of LCAO MO coefficients are thus shaped by the conditional probabilities,

$$
\begin{aligned}
& P=P\left(\chi_{A} \mid \phi_{b}\right)=\left(C_{A, b}\right)^{2}=P\left(\chi_{B} \mid \phi_{a}^{(1)}\right)=\left[C_{B, a}^{(1)}\right]^{2}, \quad \text { and } \\
& Q=P\left(\chi_{\mathrm{B}} \mid \phi_{b}\right)=\left(C_{\mathrm{B}, b}\right)^{2}=P\left(\chi_{\mathrm{A}} \mid \phi_{a}^{(1)}\right)=\left[C_{\mathrm{A}, a}^{(1)}\right]^{2}=1-P .
\end{aligned}
$$

It can be straightforwardly verified that these two MO combinations indeed satisfy the MO-orthonormality relations:

$$
\left\langle\boldsymbol{\phi}^{(1)} \mid \boldsymbol{\phi}^{(1)}\right\rangle=\mathbf{C}^{(1) \mathrm{T}}\langle\chi \mid \chi\rangle \mathbf{C}^{(1)}=\mathbf{C}^{(1) \mathrm{T}} \mathbf{C}^{(1)}=\mathbf{I},
$$

where we have used the AO orthonormality: $\langle\chi \mid \chi\rangle=\mathbf{I}$. We further assume that both atoms of the system "promolecule", consisting of the molecularly placed free atoms, 


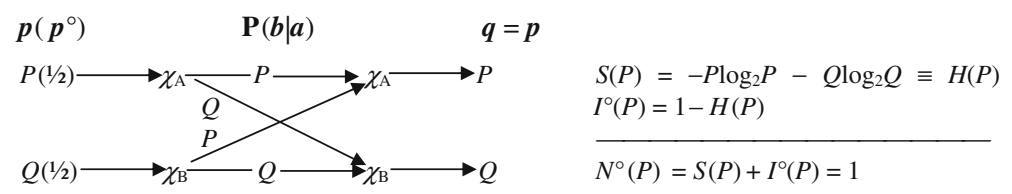

Fig. 3 Communication channel of the 2-AO model of the chemical bond and its entropy/information descriptors (in bits)

contribute a single electron each to the molecular bond system of $N=2$ electrons: $\boldsymbol{p}_{0}=(1 / 2,1 / 2)$.

Let us first examine the single-configuration description of this model chemical bond, e.g., in the HF/KS ground-state, when both (spin-paired) electrons occupy the bonding $\mathrm{MO} \phi_{b}$. The relevant $\mathrm{CBO}$ matrix $\boldsymbol{\gamma}$ reads:

$$
\boldsymbol{\gamma}=2 \boldsymbol{C}_{b} \boldsymbol{C}_{b}^{\dagger}=2\left[\begin{array}{cc}
P & \sqrt{P Q} \\
\sqrt{Q P} & Q
\end{array}\right] .
$$

It generates the following conditional probabilities of $\mathrm{AO}$ communications,

$$
\mathbf{P}(\boldsymbol{b} \mid \boldsymbol{a})=\mathbf{P}\left(\chi^{\prime} \mid \chi\right)=\left\{P(j \mid i)=\left[\begin{array}{cc}
P & Q \\
P & Q
\end{array}\right],\right.
$$

which determine the classical (probability) channel for AO communications $\chi \rightarrow \chi^{\prime}$ (Fig. 3).

In this non-symmetrical binary channel one adopts the molecular input signal, $\boldsymbol{p}=(P, Q)$, to extract the bond IT-covalency measuring the channel average communication noise [9-11]. Adopting the promolecular input signal $\boldsymbol{p}_{0}=(1 / 2,1 / 2)$, reflecting that each of the two basis functions has contributed a single electron each to form the chemical bond, allows one to determine the associated index of IT-ionicity relative to this initial, reference signal, which then measures the information capacity of this model AO channel.

The bond IT-covalency (conditional entropy $S(\boldsymbol{b} \mid \boldsymbol{a})=S(P)$ is thus determined by Binary Entropy Function (BEF) of the two complementary conditional probabilities of $\mathrm{AO}$ in $\mathrm{MO}$ [Eq. (71)], $H(P)=-P \log _{2} P-(1-P) \log _{2}(1-P)$ (see Fig. 4),

$$
S(P)=H(P)=-P \log _{2} P-Q \log _{2} Q .
$$

It exhibits the maximum value $H(1 / 2)=1$ bit for the symmetric bond $P=Q=$ $1 / 2$, e.g., the two prototype covalent bonds in chemistry: the $\sigma$ bond in $\mathrm{H}_{2}$ or the $\pi$-bond in ethylene. It vanishes for the lone-pair configurations, when $P=(0$ or 1$)$, $H(0)=H(1)=0$, marking the alternative ion-pair configurations $\mathrm{A}^{+} \mathrm{B}^{-}$and $\mathrm{A}^{-} \mathrm{B}^{+}$, respectively, relative to the initial AO occupations $\boldsymbol{n}_{0}=(1,1)$ in the assumed covalent promolecular reference, in which both atoms contribute a single electron each to form the chemical bond A-B.

The complementary mutual-information descriptor of the bond IT-ionicity (information flow) [Eq. (9)], $I_{0}(P)=1-H(P)$, which determines the channel mutual 


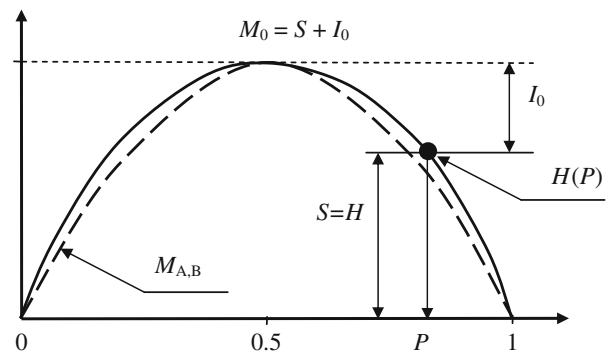

Fig. 4 Conservation of the overall entropic bond multiplicity $M_{0}(P)=1$ bit in the 2-AO model of the chemical bond, combining the conditional-entropy (average noise, bond IT covalency) $S(P)=H(P)$ and the mutual-information (information capacity, bond IT ionicity) $I_{0}(P)=1-H(P)$. In MO theory the direct bond-order of Wiberg [87] is represented by the (broken-line) parabola $M_{\mathrm{A}, \mathrm{B}}(P)=4 P(1-P) \equiv 4 P Q$

information relative to the promolecular input, is thus correctly diagnosed to reach the highest value for the two electron-transfer pairs: $I_{0}(0)=I_{0}(1)=1$ bit, and predicted to identically vanish for the purely-covalent, symmetric bond, $I_{0}(1 / 2)=0$. As explicitly shown in Fig. 4, these two components of the chemical bond multiplicity compete with one another, yielding the conserved overall IT bond index [Eq. (11)]: $M_{0}(P)=S(P)+I_{0}(P)=1$ bit, which in OCT marks the full single bond, in the whole range of the admissible bond polarizations $P \in[0,1]$.

This simple model thus properly accounts for the competition between the bond covalency and ionicity, while preserving the single bond-order measure reflected by the conserved single IT multiplicity of the chemical bond. Similar effects transpire from the quadratic bond indices formulated in the MO theory [87-97]. For example, the plot of Wiberg bond-order [87] for this model (see Fig. 4) is given by the parabola $M_{\mathrm{A}, \mathrm{B}}(P)=\left[\gamma_{A, B}(P)\right]^{2}=4 P Q=4 P(1-P)$, which closely resembles the ITcovalent plot $S(P)=H(P)$ in the same figure. It should be stressed, however, that this "complementary" indexing scheme in OCT has been designed primarily to describe the bonding patterns of the equilibrium molecular geometries. It fails to account for a decay in the overall bond multiplicity accompanying bond elongation. For the improved CItype descriptors, which remedy this shortcoming, see Ref. [45].

In this (minimum basis) 2-AO description there are three singlet configurations (of the spin-paired electrons), $\boldsymbol{\Psi}=\left(\Psi_{b}, \Psi_{n}, \Psi_{a}\right)$, including the HF/KS ground-state (bonding) configuration

$$
\Psi_{b}=\left|\phi_{b} \alpha, \phi_{b} \beta\right|,
$$

the singly-excited (S) non-bonding configuration

$$
\Psi_{n}^{(1)}=2^{-1 / 2}\left\{\left|\phi_{b} \alpha, \phi_{a}^{(1)} \beta\right|+\left|\phi_{b} \beta, \phi_{a}^{(1)} \alpha\right|\right\},
$$

and the doubly-excited (D) anti-bonding configuration

$$
\Psi_{a}^{(1)}=\left|\phi_{a}^{(1)} \alpha, \phi_{a}^{(1)} \beta\right|,
$$


into which the CI ground state is expanded in the familiar CI-[Singles and Doubles] (CISD) approximation:

$$
\Psi^{\mathrm{CI}}(2) \cong c_{b} \Psi_{b}(2)+c_{n} \Psi_{n}(2)+c_{a} \Psi_{a}(2) \equiv \Psi^{\mathrm{CISD}}(2) .
$$

The classical channel, from the probability averaging treatment, is now characterized by the ensemble average of communications $\left\{\chi_{i} \rightarrow \chi_{j}\right\}$ resulting from the internal communications $\left\{P_{i \rightarrow j}(\alpha)\right\}$ between the specified pair of AO in each configuration:

$$
\begin{aligned}
& {\left[\chi_{i}-p_{b} P_{i \rightarrow j}(b) \rightarrow \chi_{j}\right]+\left[\chi_{i}-p_{n} P_{i \rightarrow j}(n) \rightarrow \chi_{j}\right]+\left[\chi_{i}-p_{a} P_{i \rightarrow j}(a) \rightarrow \chi_{j}\right]} \\
& \equiv \chi_{i}-\left\langle P_{i \rightarrow j}\right\rangle_{\text {ens. }} \rightarrow \chi_{j}
\end{aligned}
$$

where the brackets [ ] enclose the separate internal AO propagation in each configuration $\Psi_{\alpha}$, with the weight being determined by the associated configuration probability $p_{\alpha}$.

The corresponding quantum channel, from the amplitude averaging scheme, combines the following elementary amplitudes,

$$
\begin{array}{r}
{\left[\left|\chi_{i}\right\rangle-\left\{p_{b} A_{i \rightarrow j}(b)\right\} \rightarrow\left|\chi_{j}\right\rangle\right]+\left[\left|\chi_{i}\right\rangle-\left\{p_{n} A_{i \rightarrow j}(n)\right\} \rightarrow\left|\chi_{j}\right\rangle\right]} \\
\quad+\left[\left|\chi_{i}\right\rangle-\left\{p_{a} A_{i \rightarrow j}(a)\right\} \rightarrow\left|\chi_{j}\right\rangle\right] \equiv\left|\chi_{i}\right\rangle-\left\langle A_{i \rightarrow j}\right\rangle_{a v} \rightarrow\left|\chi_{j}\right\rangle .
\end{array}
$$

They generate nine modes $\left\{P_{i \rightarrow j}(\alpha, \beta)\right\}$ of the probability scattering: three diagonal (intra-configuration) modes $\left\{P_{i \rightarrow j}(\alpha, \alpha) \equiv P_{i \rightarrow j}(\alpha)\right\}$ and six off-diagonal (interconfiguration) ones $\left\{P_{i \rightarrow j}(\alpha, \beta), \alpha \neq \beta\right\}$.

Therefore, instead of three elementary probability propagations $\left\{P_{i \rightarrow j}(\alpha)\right\}$ in the classical CI channel, one identifies nine probability modes $\left\{P_{i \rightarrow j}(\alpha, \beta)\right\}$ in the quantum CI system. While the classical communications between AO are purely intraconfiguration in character, the non-classical (quantum) network exibits both the intraand inter-configuration communications, with the latter being responsible for the quantum-mechanical interference of configuration communications.

We therefore conclude that more complex, quantum communications in molecules are predicted to be capable of a more efficient dissipation of the input AO information into the electron delocalization ("noise", orbital IT covalency), compared to the simpler classical network. One observes, however, that the interference effects, characterizing only the non-classical (amplitude) channels, can in some cases help to preserve the communication localization ("order", orbital IT ionicity), i.e., "determinicity" in molecular communications, by increasing or decreasing the AO communication probabilities relative to the associated classical network.

\section{Orbital coupling and bond multiplicities in prototype VB states}

In the 2-AO model the set of four elementary $\mathrm{AO}$ products, two ionic, $\left[\mathrm{A}^{-} \mathrm{B}^{+}\right]=$ $\chi_{\mathrm{A}}(1) \chi_{\mathrm{A}}(2)$ and $\left[\mathrm{A}^{+} \mathrm{B}^{-}\right]=\chi_{\mathrm{B}}(1) \chi_{\mathrm{B}}(2)$, and two covalent, $[\mathrm{A}-\mathrm{B}]=\chi_{\mathrm{A}}(1) \chi_{\mathrm{B}}(2)$ and 
$[\mathrm{B}-\mathrm{A}]=\chi_{\mathrm{B}}(1) \chi_{\mathrm{A}}(2)$, span the complete $t w o$-electron Hilbert space of the familiar VB approach [98]. Through the two spatial configurations of the CID expansion,

$$
\begin{aligned}
& \Psi_{b}(2)=P\left[\mathrm{~A}^{-} \mathrm{B}^{+}\right]+Q\left[\mathrm{~A}^{+} \mathrm{B}^{-}\right]+\sqrt{2 P Q} \Psi^{c o v} .(2), \\
& \Psi_{a}(2)=Q\left[\mathrm{~A}^{-} \mathrm{B}^{+}\right]+P\left[\mathrm{~A}^{+} \mathrm{B}^{-}\right]-\sqrt{2 P Q} \Psi^{c o v} .
\end{aligned}
$$

where the covalent (atomic) structure of Heitler and London represents the normalized difference of both configurations, yielding the symmetrized combination of two covalent products,

$$
\Psi^{\operatorname{cov} .}(2) \equiv 2^{-1 / 2}\left[\Psi_{b}(2)-\Psi_{a}(2)\right]=2^{-1 / 2}\{[\mathrm{~A}-\mathrm{B}]+[\mathrm{B}-\mathrm{A}]\},
$$

it is capable of representing both electronic (singlet) states of this two-electron system, e.g., the ground state,

$$
\Psi^{\mathrm{CID}}(2) \equiv \Psi_{+}(2)=c_{b} \Psi_{b}(2)+c_{a} \Psi_{a}(2), \quad\left|c_{b}\right|^{2}+\left|c_{a}\right|^{2}=1
$$

and the excited, anti-bonding combination,

$$
\Psi^{e x c} \cdot(2) \equiv \Psi_{-}(2)=-c_{a} \Psi_{b}(2)+c_{b} \Psi_{a}(2)
$$

They express all prototype chemical states of this two-electron system in VB theory. For example, for the homomonuclear (symmetric) bond, $P=Q=1 / 2$, one defines

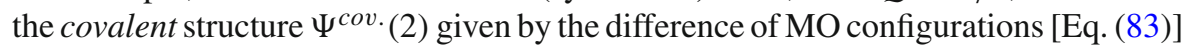
and the ionic structure $\Psi^{i o n}$. (2) representing the normalized sum of these electron configurations giving the symmetrized combination of two ionic products:

$$
\Psi^{i \text { on. }}(2) \equiv 2^{-1 / 2}\left[\Psi_{b}(2)+\Psi_{a}(2)\right]=2^{-1 / 2}\left\{\left[\mathrm{~A}^{-} \mathrm{B}^{+}\right]+\left[\mathrm{A}^{+} \mathrm{B}^{-}\right]\right\}
$$

These two reference chemical states correspond to equal configuration probabilities:

$$
\boldsymbol{p}^{i o n}=\boldsymbol{p}^{\text {cov. }}=(1 / 2,1 / 2) \equiv(1 / 2) \boldsymbol{1},
$$

thus giving rise to identical CID probability and amplitude MO-ensembles.

Therefore, both the classical (probability) and quantum (amplitude) averaging schemes do not reveal the basic chemical difference between these two prototype VB states, which is embodied in the phases of MO configurations in these two combinations. In particular, the classical averaging of the configuration conditional probabilities gives:

$$
\left\langle\mathbf{P}\left(\boldsymbol{\chi}^{\prime} \mid \boldsymbol{\chi}\right)\right\rangle_{\text {ens. }}=\mathbf{P}^{\text {cov. }}\left(\boldsymbol{\chi}^{\prime} \mid \boldsymbol{\chi}\right)=\mathbf{P}^{i o n} \cdot\left(\boldsymbol{\chi}^{\prime} \mid \boldsymbol{\chi}\right)=1 / 2\left[\mathbf{P}^{b}\left(\boldsymbol{\chi}^{\prime} \mid \boldsymbol{\chi}\right)+\mathbf{P}^{a}\left(\boldsymbol{\chi}^{\prime} \mid \boldsymbol{\chi}\right)\right]=(1 / 2) \mathbf{1}
$$


Definitions of Eqs. (83) and (86) also imply identical ensemble-average bond-orders between $\mathrm{AO}$ in both structures,

$$
\langle\boldsymbol{\gamma}\rangle_{\text {ens. }}=\sum_{\alpha=b, a} p_{\alpha} \boldsymbol{\gamma}^{\alpha}=1 / 2\left(\boldsymbol{\gamma}^{b}+\boldsymbol{\gamma}^{a}\right)=\left[\begin{array}{ll}
1 & 0 \\
0 & 1
\end{array}\right] \equiv \mathbf{I}
$$

and hence the diagonal average amplitudes of AO communications,

$$
\left\langle\mathbf{A}\left(\boldsymbol{\chi}^{\prime} \mid \boldsymbol{\chi}\right)\right\rangle_{a v .}=\mathbf{A}^{\operatorname{cov} .}\left(\boldsymbol{\chi}^{\prime} \mid \boldsymbol{\chi}\right)=\mathbf{A}^{i o n}\left(\boldsymbol{\chi}^{\prime} \mid \boldsymbol{\chi}\right)=2^{-1 / 2}\left[\mathbf{A}^{b}\left(\boldsymbol{\chi}^{\prime} \mid \boldsymbol{\chi}\right)+\mathbf{A}^{a}\left(\boldsymbol{\chi}^{\prime} \mid \boldsymbol{\chi}\right)\right]=\mathbf{I} .
$$

They generate the deterministic (diagonal) average CID channel:

$$
\left\langle\mathbf{P}\left(\boldsymbol{\chi}^{\prime} \mid \chi\right)\right\rangle_{a v .}=\left\langle\mathbf{P}^{\text {cov. }}\left(\chi^{\prime} \mid \chi\right)\right\rangle_{a v .}=\left\langle\mathbf{P}^{i o n .}\left(\chi^{\prime} \mid \chi\right)\right\rangle_{a v .}=\left\{\left[\left\langle A\left(\chi_{j} \mid \chi_{i}\right)\right\rangle_{a v}\right]^{2}\right\}=\mathbf{I} .
$$

One recalls that the two prototype VB states, $\Psi^{\operatorname{cov} .}(2)$ and $\Psi^{i o n}$. $(2)$, also represent the reference atomic and ionic dissociation limits, respectively, with the former attributing single electrons to different atoms and the latter locating both electrons on a single AO. The ionic structure indeed represents the equal participation of two admissible ion-pairs, relative to the assumed (atomic) promolecular reference. The fully coupled, classical communication system $\left\langle\mathbf{P}\left(\chi^{\prime} \mid \chi\right)\right\rangle_{\text {ens. }}=(1 / 2) \mathbf{1}$ represents in OCT the purely covalent communication system, $\left\langle S\left(\chi^{\prime} \mid \chi\right)\right\rangle_{\text {ens. }}=1$ bit and $\left\langle I_{0}\left(\chi: \chi^{\prime}\right)\right\rangle_{\text {ens. }}=0$, of the complete dissipation of the initial (input) information into the communication "noise" [11-13]. It thus reflects the maximum IT-covalency and hence the minimum IT-ionicity in this model chemical bond (see Fig. 4). It should be also observed that for the strictly deterministic average amplitude channel, $\left\langle\mathbf{P}\left(\boldsymbol{\chi}^{\prime} \mid \boldsymbol{\chi}\right)\right\rangle_{a v} .=\mathbf{I}$, the IT-covalent bond component indeed vanishes, $\left\langle S\left(\chi^{\prime} \mid \chi\right)\right\rangle_{a v} .=0$, with the complementary IT-ionic index of the channel information capacity relative to the assumed atomic promolecule, $\left\langle I_{0}\left(\chi: \chi^{\prime}\right)\right\rangle_{a v} .=1$ bit, indeed marking a single purely ionic chemical bond [11-13].

One could intuitively expect from the definition of Eq. (86) that the ionic VBstructure represents only the intra-atomic orbital communications (inter-atomically decoupled), in which the amplitude scatterings between different atoms, $\left|\chi_{A}\right\rangle \rightarrow\left|\chi_{B}\right\rangle$ and $\left|\chi_{B}\right\rangle \rightarrow\left|\chi_{A}\right\rangle$, identically vanish with only the intra-AO, fully deterministic communications $\left|\chi_{A}\right\rangle \rightarrow\left|\chi_{A}\right\rangle$ and $\left|\chi_{B}\right\rangle \rightarrow\left|\chi_{B}\right\rangle$ remaining. This conjucture is seen to be supported by the average bond-orders in this ionic state, which also reflect its average communication amplitudes of Eq. (90). The equal mixture of the $\mathrm{CBO}$ matrices for two configurations involved in $\Psi^{i o n}$. (2) thus gives the expected average amplitudes, which correctly describe the average AO populations $\left\{\left\langle n_{i}\right\rangle_{\text {ens. }}=1\right\}$ of electrons in this orbitally decoupled state, equal to those in the assumed atomic promolecule. They generate the purely deterministic average communications in this ionic reference state:

$$
\left\langle\mathbf{P}\left(\chi^{\prime} \mid \chi\right)\right\rangle_{a v}=\mathbf{I} \equiv \mathbf{P}^{i o n} \cdot\left(\chi^{\prime} \mid \chi\right) .
$$


Fig. 5 The diagonal (upper channel) and off-diagonal (lower channel) communications in two prototype VB structures

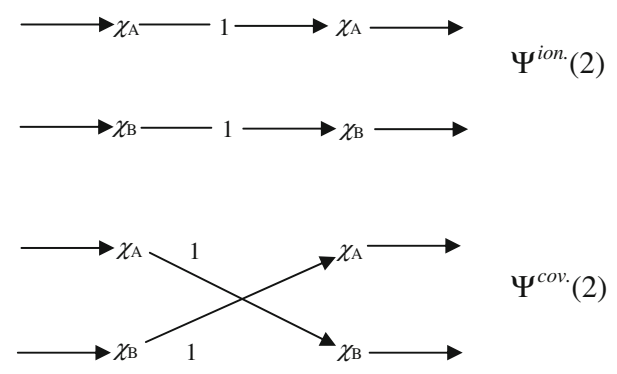

This intuitively correct prediction from the quantum (amplitude) CID MO-ensemble is in contrast to that resulting from the classical (probability) averaging scheme, which implies the full spectrum of orbital communications expected in the fully delocalized

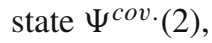

$$
\left\langle\mathbf{P}\left(\chi^{\prime} \mid \chi\right)\right\rangle_{\text {ens. }}=(1 / 2) \mathbf{1} \equiv \mathbf{P}^{\operatorname{cov} \cdot}\left(\chi^{\prime} \mid \chi\right)
$$

containing the equal mixture of both the intra- and inter-AO communications in Fig. 3.

One would also expect that the prototype covalent structure of Eq. (83) should indeed equally explore both the off-diagonal (delocalized) communications $\left|\chi_{\mathrm{A}}\right\rangle \rightarrow$ $\left|\chi_{\mathrm{B}}\right\rangle$ and $\left|\chi_{\mathrm{B}}\right\rangle \rightarrow\left|\chi_{\mathrm{A}}\right\rangle$ and the localized $\left|\chi_{\mathrm{A}}\right\rangle \rightarrow\left|\chi_{\mathrm{A}}\right\rangle$ and $\left|\chi_{\mathrm{B}}\right\rangle \rightarrow\left|\chi_{\mathrm{B}}\right\rangle$ propagations of $\Psi^{i o n}$. (2), since then the IT-covalency, reflected by the molecular conditional entropy $S\left(\chi^{\prime} \mid \chi\right)$, reaches its maximum value of 1 bit of information, while the channel ITionicity, measured by the mutual information relative to the promolecular reference, $I^{0}\left(\chi: \chi^{\prime}\right)$, identically vanishes. This expectation is indeed realized only in the classical probability averaging of Eq. (88).

Therefore, neither of the two averaging schemes in the CID MO-ensemble gives the intuitively expected predictions for both these complementary apects of the chemical bond in the 2-AO model. This analysis seems to confirm the "classical" character of the bond-ionic IT multiplicity, being correctly represented by the probability-averaging over configurations. The complementary bond-covalency appears to constitute the "quantum" aspect of the bonding pattern in a molecule, the overall IT multiplicity of which requires the amplitude-averaging over configurations.

In the alternative CID perspective [45] on the information origins of the two bond components and on the overall entropic measure of the chemical bond-order one separates the deterministic ("additive", localized) diagonal communications $\left|\chi_{\mathrm{A}}\right\rangle \rightarrow$ $\left|\chi_{\mathrm{A}}\right\rangle$ and $\left|\chi_{\mathrm{B}}\right\rangle \rightarrow\left|\chi_{\mathrm{B}}\right\rangle$, from the cross ("non-additive", delocalized) probability propagations $\left|\chi_{A}\right\rangle \rightarrow\left|\chi_{B}\right\rangle$ and $\left|\chi_{B}\right\rangle \rightarrow\left|\chi_{A}\right\rangle$ between $\mathrm{AO}$ (Fig. 5). The former are then linked to the bond ionicity effects, while the latter are ascribed to the chemical bond covalency. In this approach the overall conditional entropy generates the interactiondependent IT index of the chemical bond overall multiplicity, which correctly vanishes in the SAL and was shown to compare favourably with the Wiberg index of quantum chemistry (Fig. 4). 
As we have already observed above, the predictions of AO communications in both VB structures are the same in the given averaging scheme of the CID MO-ensemble, thus failing to distinguish the fundamental chemical difference in these two prototype bonding situations. In order to remedy this shortcoming one has to recognize the configuration phases in Eqs. (83) and (86). For the ionic structure this proposition again leads to the average amplitudes generated by the diagonal bond orders of Eq. (89),

$$
\left\langle\boldsymbol{\gamma}^{\text {ion. }}\right\rangle_{\text {ens. }}=1 / 2\left(\boldsymbol{\gamma}^{b}+\boldsymbol{\gamma}^{a}\right)=\mathbf{I}
$$

while for the covalent structure one then obtains the average CBO-difference matrix:

$$
\left\langle\boldsymbol{\gamma}^{\text {cov. }}\right\rangle_{\text {ens. }}=1 / 2\left(\boldsymbol{\gamma}^{b}-\boldsymbol{\gamma}^{a}\right)=\left[\begin{array}{ll}
0 & 1 \\
1 & 0
\end{array}\right] \equiv \mathbf{1}-\mathbf{I} \text {. }
$$

These average matrix elements (communication amplitudes) thus correctly ascribe the deterministic diagonal (intra-AO) communications $\left|\chi_{\mathrm{A}}\right\rangle \rightarrow\left|\chi_{\mathrm{A}}\right\rangle$ and $\left|\chi_{\mathrm{B}}\right\rangle \rightarrow\left|\chi_{\mathrm{B}}\right\rangle$ to $\Psi^{\text {ion. }}(2)$, while the off-diagonal (inter-AO) probability propagations $\left|\chi_{\mathrm{A}}\right\rangle \rightarrow\left|\chi_{\mathrm{B}}\right\rangle$ and $\left|\chi_{\mathrm{B}}\right\rangle \rightarrow\left|\chi_{\mathrm{A}}\right\rangle$ are identified as originating from $\Psi^{\operatorname{cov}}$. (2). This is schematically illustrated in Fig. 5. A similar partitioning of these elementary AO communications in the $\mathrm{H}_{2}$ information system has been also conjectured elsewhere [99].

In this novel perspective the $\mathrm{AO}$ communications are thus classified as the intra$\mathrm{AO}$ (ionic) or inter-AO (covalent). This conforms to the accepted chemical intuition, in which the localized AO propagations of $\Psi^{i o n .}(2)$ are exclusively associated with the bond ionicity, and the delocalized AO scatterings are solely responsible for the electron sharing (covalent) effects of the chemical bond. The same classification then applies to the conditional entropy contributions to the IT bond order: the AO diagonal terms $H($ diag. $) \equiv V_{\text {ion. }}$ are then ionic in character, the off-diagonal contribution $H$ (ndiag.) $\equiv V_{\text {cov }}$. generates a modified measure of the bond covalency, and their sum, $V=V_{\text {cov }}+V_{\text {ion. }}=S$, i.e., the overall conditional entropy of the average AO channel in the CI MO-ensemble [Eq. (93)], now stands for the overall entropic measure of the bond multiplicity.

For example, in the HF/KS 2-AO communication system (Fig. 3) probed by the molecular input probabilities $\boldsymbol{p}=(P, Q)$ one then predicts:

$$
V_{\text {ion. }}(P)=-P^{2} \log P-Q^{2} \log Q, \quad V_{\text {cov }} .(P)=-P Q[\log P+\log Q] \text {. }
$$

These two entropic components give the associated global measure of the modified bond multiplicity index, equal to $\mathrm{BEF} H(P)$,

$$
V(P)=V_{\text {cov. }}(P)+V_{\text {ion. }}(P)=(P+Q)[-P \log P-Q \log Q]=H(P) .
$$

This new index compares favourably with the Wiberg bond order in Fig. 4 and is interaction-dependent. It vanishes in the heteronuclear (ionic) dissociation limit, $\Psi^{\mathrm{CID}}(2)=\Psi^{i \text { ion. }}(2)$, at large separation between atoms, $R_{\mathrm{AB}} \rightarrow \infty$, when $(P=1$ and $Q=0)$ or $(P=0$ and $Q=1)$. This result $V_{i o n}(\infty)=0$ represents the vanishing 
noise descriptor of the first deterministic channel in Fig. 5. It should be also observed that in the symmetric case of $\mathrm{H}_{2}$ at the equilibrium separation between nuclei, when $P=Q=1 / 2$,

$$
V_{\text {ion. }}(1 / 2)=V_{\text {cov. }}(1 / 2)=1 / 2 \text { and } \quad V(1 / 2)=1 \text {. }
$$

For the spin-unrestricted (UHF/UKS) orbitals in this homonuclear (atomic) dissociation limit $R_{A B} \rightarrow \infty$, both MO represent the corresponding $\mathrm{AO}$, i.e., again $(P=1$ and $Q=0)$ or $(P=0$ and $Q=1)$ and $\Psi^{\mathrm{CID}}(2)=\Psi^{\text {cov. }}$ (2). Therefore, one again predicts $V_{\text {cov }}(\infty)=0$, the vanishing overall noise descriptor of the second deterministic channel in Fig. 5.

In a general case of the constituent atoms contributing several AO to the molecular bond system one adopts the usual atomic resolution [91], in which the sum of the intra-atomic diagonal-scattering contributions again reflects the atomic ionicity, the atom off-diagonal conditional probabilities between different $\mathrm{AO}$ generate the atomic IT covalency index, reflecting the AIM promotion in the presence of the remaining atoms, and the inter-atomic information propagation terms reflect the true chemical bonding between different atoms.

\section{Conclusion}

It has been amply demonstrated elsewhere that IT provides an attractive, novel perspective on the molecular equilibria, multiplicity, composition and localization of chemical bonds in molecular systems, and their information origins. We have presented in this analysis some new developments in CTCB covering the local information systems and the CI MO-ensemble generalization of the single-configuration OCT.

All these approaches introduce the communication perspective on several classical issues in the theory of bond multiplicities and their covalent/ionic composition. The inter-atomic conditional probabilities in HF or KS theories, generated from the bond-projected superposition principle of quantum mechanics, are proportional to the quadratic (Wiberg-type) bond indices formulated in the SCF MO description. Therefore, the strong inter-orbital communications correspond to strong Wiberg bond-order contributions. The OCT treatment of the localized (diatomic) chemical interactions in polyatomics has also been developed [11-13,69]. This approach reproduces the Wiberg bond multiplicity in diatomic molecules and allows one to resolve the overall IT bond-order of the localized bond into the associated IT covalent/ionic components. In typical polyatomic molecules these localized-bond multiplicities were also shown to closely approximate the quadratic Wiberg index of quantum chemistry, at the same time providing its IT-covalent/ionic resolution [69].

In this work OCT has been extended to cover the multi-determinantal CI-type wave functions. The effective MO-ensemble approach, which recognizes the phases of the CI configurations, has been advocated for extracting chemical interpretation from the molecular AO information channels. In the CID treatment of the 2-AO model the "diagonal" (intra-orbital) communications have been associated with the IT bond 
ionicity, while the complementary covalent component has been attributed to the "offdiagonal" (inter-orbital) probability scatterings.

It should be emphasized that the two CI MO-ensemble approaches, the classical (probability) and quantum (amplitude) averaging schemes, can be also applied to weight the local channels in each configuration used in the underlying CI expansion of the system ground state. The classical local probabilities are then calculated as the ensemble average probabilities in each configuration [see Eq. (22)]:

$$
\left\langle P_{r \rightarrow r^{\prime}}\right\rangle_{\text {ens. }}=\sum_{\alpha} p_{\alpha} P_{\boldsymbol{r} \rightarrow \boldsymbol{r}^{\prime}}(\alpha)
$$

while the associated quantum probabilities $\left\langle P_{r \rightarrow \boldsymbol{r}^{\prime}}\right\rangle_{a v} .=\left[\left\langle A_{\boldsymbol{r} \rightarrow \boldsymbol{r}^{\prime}}\right\rangle_{a v} .\right]^{2}$ are generated by the squares of the resultant average amplitudes [see Eq. (23)]:

$$
\left\langle A_{\boldsymbol{r} \rightarrow \boldsymbol{r}^{\prime}}\right\rangle_{a v}=\sum_{\alpha} p_{\alpha} A_{\boldsymbol{r} \rightarrow \boldsymbol{r}^{\prime}}(\alpha),
$$

preferably recognizing the configuration phases in the ground state:

$$
\left\langle A_{r \rightarrow r^{\prime}}\right\rangle_{a v .}=\sum_{\alpha} \operatorname{sign}\left(c_{\alpha}\right) p_{\alpha} A_{r \rightarrow r^{\prime}}(\alpha)
$$

Open Access This article is distributed under the terms of the Creative Commons Attribution License which permits any use, distribution, and reproduction in any medium, provided the original author(s) and the source are credited.

\section{References}

1. R.A. Fisher, Proc. Camb. Phil. Soc. 22, 700 (1925)

2. B.R. Frieden, Physics from the Fisher Information: A Unification, 2nd edn. (Cambridge University Press, Cambridge, 2004)

3. C.E. Shannon, Bell Syst. Tech. J. 27, 379, 623 (1948)

4. C.E. Shannon, W. Weaver, The Mathematical Theory of Communication (University of Illinois, Urbana, 1949)

5. S. Kullback, R.A. Leibler, Ann. Math. Stat. 22, 79 (1951)

6. S. Kullback, Information Theory and Statistics (Wiley, New York, 1959)

7. N. Abramson, Information Theory and Coding (McGraw-Hill, New York, 1963)

8. P.E. Pfeifer, Concepts of Probability Theory, 2nd edn. (Dover, New York, 1978)

9. R.F. Nalewajski, J. Math. Chem. 51, 297 (2013)

10. R.F. Nalewajski, Entropic Concepts in Electronic Structure Theory. Found. Chem. (in press). doi:10. 1007/s10698-012-9168-7

11. R.F. Nalewajski, Information Theory of Molecular Systems (Elsevier, Amsterdam, 2006)

12. R.F. Nalewajski, Information Origins of the Chemical Bond (Nova, New York, 2010)

13. R.F. Nalewajski, Perspectives in Electronic Structure Theory (Springer, Heidelberg, 2012)

14. R.F. Nalewajski, in Mathematical Chemistry, ed. by W.I. Hong (Nova Science Publishers, New York, 2011), pp. 247-325

15. R.F. Nalewajski, in Chemical Information and Computation Challenges in 21st Century, ed. by M.V. Putz (Nova Science Publishers, New York, 2012), pp. 61-100

16. R.F. Nalewajski, P. de Silva, J. Mrozek, in Theoretical and Computational Developments in Modern Density Functional Theory, ed. by A.K. Roy (Nova, New York, 2012), pp. 561-588 
17. R.F. Nalewajski, in Frontiers in Modern Theoretical Chemistry: Concepts and Methods (Dedicated to B. M. Deb), eds. by P.K. Chattaraj, S.K. Ghosh (Taylor \& Francis/CRC, London, 2013), pp. 143-180

18. R.F. Nalewajski, Struct. Bond. 149, 51 (2012)

19. R.F. Nalewajski, Sci. Tech. 1, 105 (2012)

20. R.F. Nalewajski, in Advances in Quantum Systems Research, ed. by Z. Ezziane (Nova, New York, 2012), in press

21. R.F. Nalewajski, in Quantum Matter Chemistry, ed. by A. Herman (American Scientific Publishers), in press

22. R.F. Nalewajski, J. Math. Chem. 51, 369 (2013)

23. R.F. Nalewajski, Ann. Phys. (Leipzig) 525, 256 (2013)

24. R.F. Nalewajski, E. Świtka, A. Michalak, Int. J. Quantum Chem. 87, 198 (2002)

25. R.F. Nalewajski, E. Świtka, Phys. Chem. Chem. Phys. 4, 4952 (2002)

26. R.F. Nalewajski, E. Broniatowska, J. Phys. Chem. A. 107, 6270 (2003)

27. R.F. Nalewajski, R.G. Parr, Proc. Natl. Acad. Sci. USA 97, 8879 (2000)

28. R.F. Nalewajski, R.G. Parr, J. Phys. Chem. A 105, 7391 (2001)

29. R.F. Nalewajski, R. Loska, Theor. Chem. Acc. 105, 374 (2001)

30. R.F. Nalewajski, Chem. Phys. Lett. 372, 28 (2003)

31. R.G. Parr, P.W. Ayers, R.F. Nalewajski, J. Phys. Chem. A 109, 3957 (2005)

32. R.F. Nalewajski, Phys. Chem. Chem. Phys. 4, 1710 (2002)

33. R.F. Nalewajski, Adv. Quantum Chem. 43, 119 (2003)

34. R.F. Nalewajski, E. Broniatowska, Theor. Chem. Acc. 117, 7 (2007)

35. F.L. Hirshfeld, Theor. Chim. Acta (Berl.) 44, 129 (1977)

36. R.F. Nalewajski, Int. J. Quantum Chem. 108, 2230 (2008)

37. R.F. Nalewajski, J. Math. Chem. 47, 667 (2010)

38. R.F. Nalewajski, P. de Silva, J. Mrozek, J. Mol. Struct: THEOCHEM 954, 57 (2010)

39. R.F. Nalewajski, A.M. Köster, S. Escalante, J. Phys. Chem. A 109, 10038 (2005)

40. A.D. Becke, K.E. Edgecombe, J. Chem. Phys. 92, 5397 (1990)

41. B. Silvi, A. Savin, Nature 371, 683 (1994)

42. A. Savin, R. Nesper, S. Wengert, T.F. Fässler, Angew. Chem. Int. Ed. Engl. 36, 1808 (1997)

43. R.F. Nalewajski, J. Phys. Chem. A 104, 11940 (2000)

44. R.F. Nalewajski, J. Math. Chem. 49, 2308 (2011)

45. R.F. Nalewajski, J. Math. Chem. 51, 7 (2013)

46. R.F. Nalewajski, K. Jug, in Reviews of Modern Quantum Chemistry: A Celebration of the Contributions of Robert G. Parr, vol. I, ed. by K.D. Sen (World Scientific, Singapore, 2002), p. 148

47. R.F. Nalewajski, Struct. Chem. 15, 391 (2004)

48. R.F. Nalewajski, Mol. Phys. 102, 531, 547 (2004)

49. R.F. Nalewajski, Mol. Phys. 103, 451 (2005)

50. R.F. Nalewajski, Mol. Phys. 104, 1977, 2533 (2006)

51. R.F. Nalewajski, Theor. Chem. Acc. 114, 4 (2005)

52. R.F. Nalewajski, J. Math. Chem. 38, 43 (2005)

53. R.F. Nalewajski, J. Math. Chem. 43, 265 (2008)

54. R.F. Nalewajski, J. Math. Chem. 45, 607 (2009)

55. R.F. Nalewajski, J. Math. Chem. 45, 709 (2009)

56. R.F. Nalewajski, J. Math. Chem. 45, 776 (2009)

57. R.F. Nalewajski, J. Math. Chem. 45, 1041 (2009)

58. R.F. Nalewajski, Int. J. Quantum Chem. 109, 425, 2495 (2009)

59. R.F. Nalewajski, J. Math. Chem. 47, 709 (2010)

60. R.F. Nalewajski, J. Math. Chem. 49, 592 (2011)

61. R.F. Nalewajski, J. Math. Chem. 43, 780 (2008)

62. R.F. Nalewajski, J. Phys. Chem. A 111, 4855 (2007)

63. R.F. Nalewajski, Mol. Phys. 104, 3339 (2006)

64. R.F. Nalewajski, J. Phys. Chem A 107, 3792 (2003)

65. R.F. Nalewajski, Mol. Phys. 104, 255 (2006)

66. R.F. Nalewajski, Ann. Phys. (Leipzig) 13, 201 (2004)

67. H.B. Callen, Thermodynamics: An Introduction to the Physical Theories of the Equilibrium Thermostatics and Irreversible Thermodynamics (Wiley, New York, 1960)

68. R.F. Nalewajski, Adv. Quantum Chem. 56, 217 (2009) 
69. R.F. Nalewajski, D. Szczepanik, J. Mrozek, Adv. Quantum Chem. 61, 1 (2011)

70. R.F. Nalewajski, D. Szczepanik, J. Mrozek, J. Math. Chem. 50, 1437 (2012)

71. R.F. Nalewajski, J. Math. Chem. 47, 692, 808 (2010)

72. R.F. Nalewajski, J. Math. Chem. 49, 806 (2011)

73. R.F. Nalewajski, J. Math. Chem. 49, 371, 546 (2011)

74. R.F. Nalewajski, P. Gurdek, J. Math. Chem. 49, 1226 (2011)

75. R.F. Nalewajski, Int. J. Quantum Chem. 112, 2355 (2012)

76. R.F. Nalewajski, P. Gurdek, Struct. Chem. 23, 1383 (2012)

77. R.F. Nalewajski, Int. J. Quantum Chem. 113, 766 (2013)

78. S. López-Rosa, Information-Theoretic Measures of Atomic and Molecular Systems, PhD Thesis, University of Granada 2010

79. S. López-Rosa, R.O. Esquivel, J.C. Angulo, J. Antolín, J.S. Dehesa, N. Flores- Gallegos, J. Chem. Theory Comput. 6, 145 (2010)

80. H. Shull, J. Am. Chem. Soc. 82, 1287 (1960)

81. H. Shull, J. Am. Chem. Soc. 86, 1469 (1964)

82. H. Shull, J. Phys. Chem. 66, 2320 (1962)

83. S. Hagstrom, H. Shull, Rev. Mod. Phys. 35, 624 (1963)

84. P.A.M. Dirac, The Principles of Quantum Mechanics, 4th edn. (Clarendon, Oxford, 1958)

85. R.F. Nalewajski, On Phases and Interference of Local Communications in Molecules, J. Math. Chem. (in press)

86. R.F. Nalewajski, J. Math. Chem. 44, 414 (2008)

87. K.A. Wiberg, Tetrahedron 24, 1083 (1968)

88. M.S. Gopinathan, K. Jug, Theor. Chim. Acta (Berl.) 63, 497, 511 (1983)

89. K. Jug, M.S. Gopinathan, in Theoretical Models of Chemical Bonding, Vol. II, ed. by Z.B. Maksić, (Springer, Heidelberg, 1990), p. 77.

90. I. Mayer, Chem. Phys. Lett. 97, 270 (1983)

91. R.F. Nalewajski, A.M. Köster, K. Jug, Theor. Chim. Acta (Berl.) 85, 463 (1993)

92. R.F. Nalewajski, J. Mrozek, Int. J. Quantum Chem. 51, 187 (1994)

93. R.F. Nalewajski, S.J. Formosinho, A.J.C. Varandas, J. Mrozek, Int. J. Quantum Chem. 52, 1153 (1994)

94. R.F. Nalewajski, J. Mrozek, G. Mazur, Can. J. Chem. 100, 1121 (1996)

95. R.F. Nalewajski, J. Mrozek, A. Michalak, Int. J. Quantum Chem. 61, 589 (1997)

96. J. Mrozek, R.F. Nalewajski, A. Michalak, Polish J. Chem. 72, 1779 (1998)

97. R.F. Nalewajski, Chem. Phys. Lett. 386, 265 (2004)

98. W. Heitler, F. London, Z. Physik 44, 455 (1927)

99. R.F. Nalewajski, Mol. Phys. 104, 365 (2006) 